
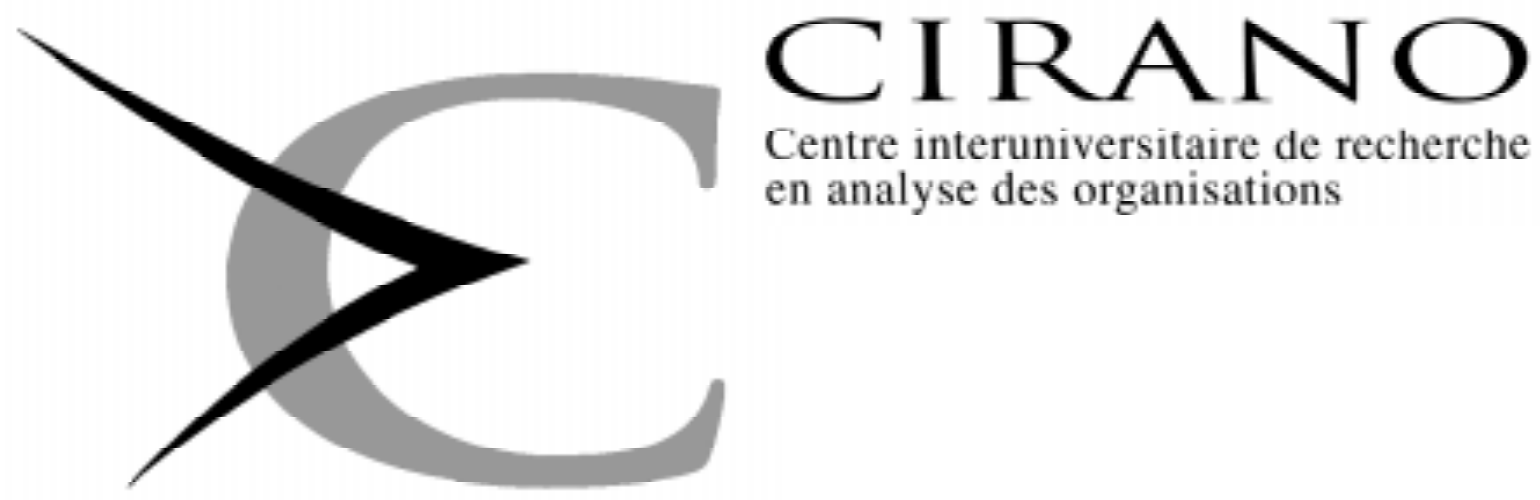

Centre interuniversitaire de recherche en analyse des organisations

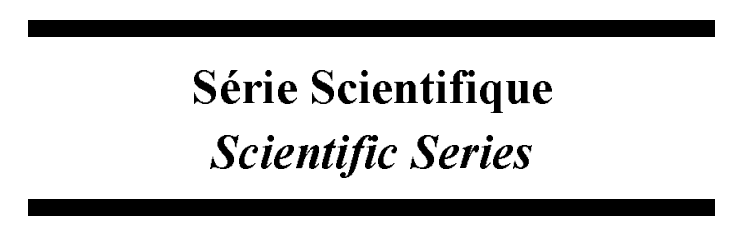

$\mathrm{N}^{\circ}$ 95s-22

CONSENSUS, DISPERSION ET PRIX DES TITRES

Jean-François L'Her, Jean-Marc Suret 


\section{CIRANO}

Le CIRANO est une corporation privée à but non lucratif constituée en vertu de la Loi des compagnies du Québec. Le financement de son infrastructure et de ses activités de recherche provient des cotisations de ses organisations-membres, d'une subvention d'infrastructure du ministère de l'Industrie, du Commerce, de la Science et de la Technologie, de même que des subventions et mandats obtenus par ses équipes de recherche. La Série Scientifique est la réalisation d'une des missions que s'est données le CIRANO, soit de développer l'analyse scientifique des organisations et des comportements stratégiques.

CIRANO is a private non-profit organization incorporated under the Québec Companies Act. Its infrastructure and research activities are funded through fees paid by member organizations, an infrastructure grant from the Ministère de l'Industrie, du Commerce, de la Science et de la Technologie, and grants and research mandates obtained by its research teams. The Scientific Series fulfils one of the missions of CIRANO: to develop the scientific analysis of organizations and strategic behaviour.

Les organisations-partenaires / The Partner Organizations

-Ministère de l'Industrie, du Commerce, de la Science et de la Technologie.

-École des Hautes Études Commerciales.

-École Polytechnique.

- Université de Montréal.

-Université Laval.

-McGill University.

-Université du Québec à Montréal.

-Bell Québec.

- La Caisse de dépôt et de placement du Québec.

-Hydro-Québec.

-Banque Laurentienne du Canada.

-Fédération des caisses populaires de Montréal et de 1'Ouest-du-Québec.

-Téléglobe Canada.

-Société d'électrolyse et de chimie Alcan Ltée.

-Avenor

Ce document est publié dans l'intention de rendre accessible les résultats préliminaires de la recherche effectuée au CIRANO, afin de susciter des échanges et des suggestions. Les idées et les opinions émises sont sous l'unique responsabilité des auteurs, et ne représentent pas nécessairement les positions du CIRANO ou de ses partenaires.

This paper presents preliminary research carried out at CIRANO and aims to encourage discussion and comment. The observations and viewpoints expressed are the sole responsibility of the authors. They do not necessarily represent positions of CIRANO or its partners.

ISSN 1198-8177 


\title{
Consensus, dispersion et prix des titres ${ }^{\star}$
}

\author{
Jean-François L'Her', Jean-Marc Surett
}

\begin{abstract}
Résumé
Cette étude montre qu'il est possible, dans un cadre d'équilibre d'anticipations rationnelles bruité, d'établir une relation linéaire formelle entre le prix des titres, la moyenne (le consensus) et la dispersion des anticipations des agents. Les variations de la moyenne et de la dispersion des anticipations des agents dues à l'arrivée d'information ont respectivement un effet positif et négatif sur le prix des titres. Les anticipations des agents sont mesurées par les prévisions de bénéfices réalisées par les analystes financiers et transmises par $I / B / E / S$. Les difficultés soulevées par cette estimation, ainsi que les dimensions institutionnelles de l'industrie de l'analyse financière sont examinées. Les principaux résultats sont les suivants : 1) Les variations les plus importantes du consensus (en valeur absolue) correspondent aux variations les plus importantes (en valeur absolue) de la dispersion des prévisions d'analystes. 2) Les changements dans le consensus et la dispersion des prévisions sont liés positivement et négativement aux rendements des actions canadiennes. En raison du décalage entre la réalisation et la diffusion des prévisions, une part importante de l'ajustement de prix a lieu avant la diffusion publique des changements dans les prévisions. 3) L'effet des variations du consensus sur le rendement des titres domine l'effet des variations de la dispersion des prévisions. Les révisions du consensus à la hausse associées aux bonnes nouvelles ont un impact plus important sur le prix lorsqu'elles s'accompagnent d'une réduction de la dispersion. Inversement, les mauvaises nouvelles ont un impact plus fort si elles augmentent la dispersion des anticipations. Il semble donc que la compréhension de l'impact de l'arrivée d'information sur le prix des titres ne dépende pas uniquement du fait que les anticipations sont corrigées à la hausse (bonne nouvelle) ou à la baisse (mauvaise nouvelle), c'est-à-dire du sens et de l'ampleur de la révision moyenne des anticipations, mais aussi de son impact sur la dispersion des anticipations.
\end{abstract}

Mots clés : modèle d'équilibre d'anticipations rationnelles bruité, hétérogénéité des anticipations, consensus, dispersion, prévisions des analystes financiers, prix des titres.

* Cette recherche a été financée par le Conseil Canadien de Recherche en Sciences Humaines et par le Fonds FCAR. Les auteurs remercient $\mathrm{I} / \mathrm{B} / \mathrm{E} / \mathrm{S}$ qui a fourni les prévisions d'analystes nécessaires à l'étude et Pierre Lemieux pour son aide dans le traitement des données.

${ }^{\dagger}$ École des Hautes Études Commerciales de Montréal.

${ }^{\ddagger}$ Centre Interuniversitaire de Recherche en Analyse des Organisations (CIRANO), Montréal et Centre de Recherche en Économie et Finance Appliquées, (CRÉFA) Université Laval, Ste Foy, (Québec), G1K 7P4, (41865671 34). 


\section{CONSENSUS, DISPERSION ET PRIX DES TITRES}

La valeur d'une action dépend des anticipations des investisseurs quant aux flux monétaires futurs qu'elle génèrera. Les prévisions de bénéfice jouent un rôle important dans la formation de ces anticipations. Brown, Foster et Noreen (1985), Brown, Richardson et Trzcinka, (1991) et L'Her et Suret (1991) ont montré que les changements dans la prévision moyenne (le consensus) sont associés positivement aux rendements des actions. Mais qu'en est-il de la relation entre les changements dans la dispersion des prévisions et le rendement des titres ? Cette étude montre de manière analytique et empirique que le prix des titres est lié à la moyenne (le consensus), mais

également à la dispersion des anticipations. Nous situons tour à tour les contributions théorique et empirique de l'étude par rapport aux études antérieures.

Sur le plan théorique, l'article se situe dans le courant de recherche qui reconnaît que les agents ont des anticipations hétérogènes et que les prix des titres jouent un rôle informatif ; il utilise donc un modèle d'équilibre d'anticipations rationnelles partiellement révélateur (ou bruité). Ces modèles intègrent les activités d'acquisition d'information des agents, l'hétérogénéité de leurs anticipations et prêtent un rôle informatif aux prix des titres dans la mesure où ils révèlent aux agents moins informés l'information privée détenue par les agents mieux informés. Ces modèles permettent de mieux rendre compte des relations qui lient arrivée d'information (publique ou privée), formation des anticipations des agents, volumes de transactions et prix des titres. Jusqu'à maintenant, les articles se sont intéressés à l'impact de l'arrivée d'information, généralement l'annonce des bénéfices, sur la formation des anticipations des agents et sur le prix des titres (Holthausen et Verrecchia, 1990 ; Abarbanel, Lanen et Verrecchia, 1994) ou les volumes de transactions (Kim et Verrecchia, 1991a, 1991b). L'étude proposée se distingue des travaux antérieurs dans le sens où elle met l'accent sur les effets des changements dans la distribution des anticipations des agents sur le rendement des titres. Ces changements sont considérés comme la conséquence de l'arrivée d'information non anticipée. Cette information a trait à l'économie, au secteur d'activité ou à l'entreprise, mais n'est pas identifiée.

Dans un modèle d'équilibre en attentes rationnelles bruité, l'hétérogénéité des anticipations des investisseurs subsiste à l'équilibre. Le prix des titres est alors d'autant plus faible que la dispersion des anticipations des agents est importante (Kazemi, 1991). Cette conclusion rejoint celle d'un autre courant de recherche qui considère que le risque d'estimation lié à la divergence dans les anticipations des agents doit être rétribué. L'hétérogénéité des anticipations est assimilée à une mesure 
de risque ex ante (Arbel et Strebel, 1982, Barry et Brown, 1985 ; Cragg et Malkiel, 1982) ou à une mesure de l'information incomplète (Merton, 1987). Nous sommes donc en présence de travaux divers qui, en utilisant des cadres de références différents, concluent généralement à une relation inverse entre la dispersion des anticipations et les prix des actions. Ils ne proposent toutefois pas d'expressions vérifiables liant les prix, l'anticipation moyenne et la dispersion. La première contribution de l'article est donc l'élaboration d'une telle expression, qui soit empiriquement vérifiable et qui réconcilie les divers cadres d'analyse possibles.

Sur le plan empirique, quelques études ont analysé la relation entre le prix des titres et la dispersion des anticipations, généralement mesurée par la dispersion des prévisions de bénéfices réalisées par les analystes financiers. Barry et Jennings, (1992) n'observent aucun effet de la dispersion des anticipations sur les prix. La dispersion et ses changements influenceraient plutôt les volumes de transactions (Ajinkya, Atiase et Gift, 1991). Toutefois, certains auteurs concluent que le marché offre une prime pour le risque d'estimation ou d'information incomplète lié à la dispersion des anticipations (Peterson et Peterson, 1982 ; Givoly et Lakonishok ; 1988 ; Amihud et Mendelson, 1989). Ils vérifient donc la relation théorique énoncée plus haut. Ces divers travaux considèrent que les variations dans la dispersion des anticipations sont des événements isolés. Or, Holthausen et Verrecchia (1990), entre autres, montrent que ces changements coïncident souvent avec une modification importante des anticipations moyennes. Les variations de la dispersion et des anticipations moyennes doivent donc être étudiées simultanément. C'est ce que propose cette étude.

Finalement, l'analyse de la relation entre la dispersion des anticipations et le prix des titres a également des implications pratiques importantes. En effet, si la réduction de l'hétérogénéité des anticipations provoque un accroissement des prix, la divulgation d'information propre à établir un plus grand consensus entre les agents doit devenir un objectif important lors de la préparation des états financiers (Morse, Stephan et Stice, 1991 ; Brown et Han, 1992). L'effet négatif de l'annonce d'une information défavorable pourrait être limité si l'information transmise à cette occasion est de nature à faire converger les prévisions des agents. Inversement, l'effet positif d'une annonce favorable pourrait être amplifié si cette annonce réduit la divergence des anticipations des agents. Enfin, une meilleure connaissance de cette relation permettrait de mieux spécifier les travaux empiriques qui analysent les effets d'annonce sans, le plus souvent, tenir compte des conséquences de ces annonces sur la dispersion des anticipations des agents. 
Ces diverses justifications nous conduisent donc à examiner s'il existe une relation significative entre les rendements des actions canadiennes et les changements dans le consensus et dans la dispersion des anticipations de prix des agents. Une telle étude soulève trois problèmes. En premier lieu, il faut établir un lien conceptuel entre les trois variables. Nous proposons donc, dans une première section, une forme fonctionnelle de la relation dans le cadre d'un modèle d'équilibre en attentes rationnelles bruité. En second lieu, il faut estimer le consensus et la dispersion des anticipations des agents. Ils sont mesurés par les prévisions des analystes financiers distribuées par I/B/E/S (Institutional Brokers Estimate System). Les difficultés posées par cette estimation (Abarbannel, Lanen et Verrecchia, 1994), ainsi que les caractéristiques des prévisions $I / B / E / S$ au Canada sont analysées dans la seconde section du texte qui propose en outre une description des aspects institutionnels des prévisions de bénéfice au Canada. Enfin, la troisième section présente la vérification empirique de la relation établie sur le plan conceptuel, à l'aide de deux méthodes complémentaires. Il s'agit d' une étude associative basée sur des modèles de régressions et une analyse de type résiduel basée sur des portefeuilles construits sur la base de changements dans la distribution des prévisions des analystes financiers. La sensibilité des résultats aux divers choix de méthodes, d'indices et de modèles est systématiquement vérifiée et les variables de contrôle suggérées par Abarbannel, Lanen et Verrecchia (1994) sont prises en compte.

\section{CADRE CONCEPTUEL}

Dans une première étape, nous donnons une expression générale du prix d'un actif risqué dans le cadre d'un modèle d'équilibre d'anticipations rationnelles partiellement révélateur ou bruité (ÉARB par la suite). Dans une seconde étape, cette expression est modifiée et le prix de l'actif risqué est alors fonction de l'anticipation moyenne des agents (le consensus) et de l'hétérogénéité des anticipations des agents (la dispersion). Dans une dernière étape, cette équation est transformée de telle sorte qu'elle puisse être testée empiriquement. Les dérivations sont reportées en annexe (équations A1 à A23) de manière à alléger le texte. 


\subsection{Une expression du prix d'équilibre}

Nous considérons une économie de pur échange à une période dans laquelle existent deux actifs, l'un sans risque et l'autre risqué. Leurs prix actuels sont respectivement 1 et $P$. Leurs prix de fin de période sont quant à eux égaux à $R=1+R_{f}$ (où $R_{f}$ représente le taux d'intérêt sans risque) et $\tilde{C}$. Les agents se divisent en 2 classes : les agents informés et les agents non informés (noise traders ou liquidity traders). Les premiers sont une infinité dénombrable. Ils maximisent l'utilité espérée de leur patrimoine futur étant donné le prix actuel de l'actif risqué, $P$, et la réalisation, $Y_{j}$,d'une variable aléatoire $\tilde{Y}_{j}=\tilde{C}+\tilde{e}_{j}$ qui représente de l'information privée relative à la valeur finale de l'actif risqué $\tilde{C}{ }^{2}$. La moyenne et l'écart type non conditionnels de $\tilde{C}$ et $\tilde{e}_{j}$ sont respectivement égaux à $\bar{C}$ et $\sigma_{c}$, puis $0, \sigma_{e}$. De plus on a $E\left(\tilde{e}_{j} \mid \tilde{C}\right)=0$ et $E\left(\tilde{e}_{j} \mid \tilde{e}_{\tilde{k}}\right)=0$ pour $\mathrm{j} \neq \mathrm{k}$. Bien qu'ils connaissent la distribution conjointe des variables $\left(Y_{j}, \tilde{P}, \tilde{C}\right)$, les investisseurs informés ne peuvent inférer la vraie valeur de l'actif risqué, en raison de la demande aléatoire des investisseurs non informés, notée $\tilde{Z} \sim\left(\bar{Z}, \sigma_{z}\right)$ qui introduit une source de bruit. En effet, les investisseurs non informés échangent des titres pour des raisons autres que celles de nature spéculative, essentiellement pour des besoins de consommation immédiate. Le système de prix est alors bruité dans la mesure où il ne révèle que partiellement l'information aux investisseurs informés. Si l'on note $S^{T}$ l'offre totale d'actif risqué, l'offre disponible pour les investisseurs informés n'est plus que $\tilde{S}=\left(S^{T}-\tilde{Z}\right)$ (avec $\tilde{S} \sim N\left(\bar{S}, \sigma_{s}=\sigma_{z}\right)$ ).

Sous la double hypothèse que les fonctions d'utilité des investisseurs sont de la forme exponentielle négative et que le prix de l'actif risqué est distribué normalement, la demande d'un investisseur informé notée $Z_{j}$ sera égale à (A1 et A2) :

$$
Z_{j}=r_{j} \cdot \frac{E\left(\tilde{C} \mid Y_{j}, P\right)-R P}{\operatorname{Var}\left(\tilde{C} \mid Y_{j}, P\right)}=r_{j} \cdot \frac{f_{j}-R P}{V}
$$

\footnotetext{
${ }^{1}$ Le cadre d'analyse est similaire à celui décrit par Holthausen et Verrecchia (1990). Toutefois, il s'agit ici d'un modèle monopériodique et non d'un modèle à deux périodes dans lequel intervient l'arrivée d'information.

${ }^{2}$ Le fait que $Y$ et $e$ soient indicés par $j$ indique que ces variables sont spécifiques à l'investisseur. L'hétérogénéité des anticipations découle ici de différences dans l'information privée et non simplement de différences dans le traitement de l'information.
} 
où $r_{j}$ représente la tolérance au risque des investisseurs, alors que $f_{j}$ et $V$ désignent l'espérance et la variance conditionnelles du prix de l'actif risqué sachant l'information privée, $Y_{j}$ et le prix actuel de l'actif risqué, $P$. A l'équilibre, la demande agrégée des investisseurs informés égale l'offre nette stochastique et le prix d'équilibre de l'actif risqué peut s'exprimer comme suit (A4 à A7) :

$$
P=\frac{r B_{0}}{r\left(R-B_{2}\right)}+\frac{B_{1}}{R-B_{2}} \tilde{C}-\frac{V}{r\left(R-B_{2}\right)} \tilde{S}
$$

où $B_{0}, B_{1}, B_{2}$ et $V(\mathrm{~A} 8$ à $\mathrm{A} 11)$ sont fonction des paramètres $\sigma_{c}, \sigma_{e}, \sigma_{s}, \mathrm{r}, \bar{C}, \bar{S}$ et $r$ représente la mesure agrégée de la tolérance au risque. Le prix de l'actif risqué est donc une fonction linéaire négative de l'offre d'actif risqué $\tilde{S}$ et une fonction positive de la valeur de fin de période de l'actif risqué, $\tilde{C}$. Celle-ci n'est pas observable, mais comme les investisseurs formulent individuellement des anticipations à son sujet, il est possible de réécrire le prix de l'actif risqué en fonction de l'anticipation moyenne des agents et de la dispersion des anticipations autour de cette moyenne. La section suivante formalise ces relations, en s'inspirant de Kazemi (1991).

\subsection{Anticipation moyenne, dispersion des anticipations et prix des titres}

- Anticipation moyenne : Dans le cadre d'un modèle d'ÉARB, il est possible d'exprimer l'anticipation moyenne, $A M$, des investisseurs informés (moyenne des espérances conditionnelles du prix de l'actif risqué étant donné une information privée et le prix de l'actif risqué) comme une fonction linéaire du prix actuel et du prix futur de 1'actif risqué (A12 et A13):

$$
A M=B_{0}+B_{1} \tilde{C}+B_{2} P
$$

- Dispersion : De la même façon, il est possible d'exprimer la dispersion des anticipations, DISP, comme étant l'écart type de la distribution des anticipations individuelles autour du consensus. Elle est proportionnelle à $B_{1}$ (A14 et A15) :

$$
D I S P=B_{1} \sigma_{e}
$$

Il est donc maintenant possible de transformer l'équation (2), pour obtenir une nouvelle expression du prix d'équilibre de l'actif risqué (A16 à A20) : 


$$
P=\frac{A M}{R}-\left(\frac{\tilde{S} \sigma_{e}}{r R}\right) D I S P
$$

Cette expression formalise une relation entre l'anticipation moyenne, la dispersion des anticipations et le prix des titres. Le prix d'équilibre de l'actif risqué est relié positivement à l'anticipation moyenne des investisseurs $(A M)$ et négativement à la dispersion des anticipations individuelles.

\subsection{Modèle empirique}

La dernière étape de la démarche consiste à transformer l'équation (5), pour en extraire une forme permettant des tests empiriques. Il suffit pour cela de mettre en évidence le rendement comme variable expliquée (A21 à A23) :

$$
\frac{P_{t}-P_{t-1}}{P_{t-1}}=R_{t}=\alpha_{0}+\alpha_{1} \frac{\Delta A M}{P_{t-1}}+\alpha_{2} \frac{\Delta D I S P}{P_{t-1}}+\varepsilon
$$

où $\triangle A M$ et $\triangle D I S P$ représentent respectivement la révision de l'anticipation moyenne et le changement dans la dispersion des anticipations des agents. L'équation (6) représente une forme opérationnelle de l'hypothèse générale énoncée en introduction. Avant de passer aux tests empiriques de cette relation, il faut toutefois définir et analyser les mesures de la moyenne et de la dispersion des anticipations des agents. C'est l'objectif de la section suivante.

2

PROBLÈMES DE MESURE ET DESCRIPTION DES DONNÉES

Dans cette section, nous discutons tout d'abord les problèmes que soulève la mesure des anticipations des agents. Nous présentons par la suite l'échantillon utilisé, ainsi que les mesures des changements dans le consensus et la dispersion. Enfin, nous analysons l'évolution de ces variables sur la période étudiée. 


\subsection{Mesure des anticipations des agents}

La recherche empirique utilise couramment les prévisions de bénéfices comptables produites par les analystes financiers ( $P A F$, par la suite) pour estimer les anticipations de prix des agents. L'écart type et le coefficient de variation des $P A F$ sont donc deux mesures courantes de la dispersion des anticipations des investisseurs (Morse, Stephan et Stice, 1991 ; Stickel, 1991 ; Atiase et Bamber, 1994). Abarbannel, Lanen et Verrecchia (ALV, 1994) montrent toutefois que les $P A F$ sont des mesures imparfaites des anticipations des investisseurs individuels pour au moins trois raisons. En premier lieu, les analystes disposent de sources d'information communes auxquelles les investisseurs individuels n'ont pas nécessairement accès. Le recours à ces mêmes sources provoque une erreur de prévision commune à tous les analystes. La dispersion de leurs prévisions peut donc différer de celle des anticipations des investisseurs. En second lieu, les agents formulent leurs anticipations à partir des $P A F$, mais ils disposent aussi d'autres sources d'information privées. L'écart entre leurs anticipations et les $P A F$ sera donc une fonction de l'importance relative de ces sources privées. Finalement, les modèles sont généralement établis en termes de prix futurs qu'anticipent les agents, alors que les analystes produisent des prévisions de bénéfices. Négliger l'effet de ces décalages entre les prévisions des analystes et les anticipations des agents conduit par conséquent à des erreurs de mesure.

L'estimation de l'anticipation moyenne par la moyenne ou la médiane des prévisions semble poser relativement peu de problèmes. En effet, ALV écrivent (p. 8) que "when analysts' individual forecasts are given equal weight, as assumed here and implicitely, in most empirical studies, the mean forecast is a sufficient statistic for the

information in the collection of forecasts". Cependant, l'estimation de l'hétérogénéité des anticipations par la dispersion des $P A F$ pose plus de problèmes. ALV montrent (p.8) que la précision des anticipations de rendements que formulent les investisseurs à la suite d'une annonce de bénéfice est fonction de 4 variables. La première est l'importance de l'erreur commune à tous les analystes, la seconde est la précision de l'annonce, la troisième est le nombre d'analystes qui formulent des prévisions et la quatrième est la dispersion des prévisions de ces analystes. La dispersion des prévisions des analystes ne capture donc pas tous les éléments qui influencent la précision des anticipations des investisseurs. Comme le notent ALV, une spécification correcte des modèles empiriques demande que l'on tienne compte des autres dimensions. Plus précisément, ils écrivent (p.27) : "to ensure that dispersion adequately reflects total forecast precision in cross-sectional tests, 
controls for the number of analysts contributing to the consensus and the amount of common information embedded in forecasts are required" 3 .

Les tests des hypothèses découlant de l'équation (6) devront tenir compte du nombre d'analystes qui formulent des prévisions pour chacun des titres. Par ailleurs, l'estimation de l'importance relative de l'information commune sera estimée, comme le proposent Atiase et Bamber (1994), par la dispersion des prévisions des analystes au début du mois où l'on observe le changement de la distribution des anticipations. Ces auteurs montrent en effet que cette dispersion peut être utilisée comme a proxy for the unobservable theoretical predisclosure information asymetry construct. Les tests empiriques menés par la suite sont basés sur les prévisions d'analystes fournies par the Institutional Brokers Estimate System $(I / B / E / S)$. Celles-ci ont été jusqu'à maintenant peu utilisées au Canada. Nous décrirons donc sommairement l'origine, le traitement et l'évolution de ces données.

\subsection{Les prévisions d'analystes financiers au Canada}

- Description générale : Au Canada, le service développé par Lynch, Jones et Ryan fournit, comme aux États-Unis, 2 types de prévisions : des données individuelles, c'est-à-dire le détail des prévisions réalisées par chacun des analystes, et des données agrégées, portant sur la distribution des prévisions. Les données agrégées, les plus souvent utilisées en recherche, comprennent pour l'exercice en cours et pour le suivant : la moyenne, la médiane ou l'écart type des prévisions de bénéfice par action (BPA), l'estimation la plus basse et l'estimation la plus élevée, le nombre d'analystes financiers suivant le titre, le nombre de révisions à la hausse et à la baisse, le ratio cours/bénéfice moyen anticipé et le mois de fin d'exercice financier. Ces données sont identiques à celles fournies aux États-Unis et ont été très souvent décrites (voir, par exemple, Brown, Foster et Noreen, 1985). Nous nous limitons donc ici aux spécificités canadiennes. Elles sont récapitulées au tableau 1 qui présente un sommaire portant sur le nombre de titres suivis et sur le nombre de $P A F$ disponibles en décembre de chacune des années.

\footnotetext{
${ }^{3}$ D'après la modélisation de ALV, la précision de l'annonce devrait être prise en compte. Toutefois, dans cette étude, nous n'étudions pas l'arrivée d'une information. Nous observons directement les effets de l'arrivée de l'ensemble des informations sur la distribution des anticipations. Nous faisons donc l'hypothèse qu'il existe une relation monotone entre la précision des annonces faites pendant un mois donné et le changement de la dispersion des $P A F$.
} 
De 1976 à $1982, I / B / E / S$ ne fournissait de prévisions que pour une cinquantaine de titres, suivis en moyenne par 3 à 5 analystes. Le nombre de titres suivis s'est ensuite rapidement accru pour atteindre 330 en 1985. Le nombre moyen de prévisions par titre est alors passé à 8 . Ce n'est donc qu'à partir de cette date que les prévisions canadiennes deviennent utilisables dans les études empiriques. Le nombre de titres suivis a diminué de façon importante, passant de 490 en 1988 à 402 en 1992. Dans le même temps, le nombre de prévisions a baissé de 3712 à 2566 (pour les mois de décembre). Il est vraisemblable que cette baisse de la participation des courtiers canadiens à $L / B / E / S$ trouve sa source dans l'importante restructuration qu'a subie cette industrie durant cette période. Alors que le nombre de titres suivis et celui des prévisions tendent à se redresser depuis 1992, le nombre moyen d'analystes par titre a baissé pour se situer à 6.5. Au mois de mai 1994, 458 titres étaient suivis et 2985 prévisions étaient disponibles. La couverture moyenne de 6.5 analystes par titre cache d'importantes différences, illustrées par la répartition des titres suivant leur couverture. Environ $15 \%$ des titres ne sont suivis que par un seul analyste, alors que $38 \%$ de ces titres sont suivis par un nombre d'analystes variant de 2 à 5 . Les titres largement suivis (6 analystes et plus) représentent donc moins de la moitié de l'ensemble. Il n'existe qu'une faible proportion (environ $15 \%$ ) des titres qui intéressent plus de 12 analystes. L'activité des analystes financiers canadiens est donc largement concentrée et la majorité des efforts d'analyse portent sur près de 70 titres. Sur le plan de la recherche empirique, cette situation implique l'élimination d'une partie importante de l'échantillon, puisqu'on considère généralement que les mesures de dispersion n'ont guère de signification lorsque le titre est suivi par moins de 6 analystes.

L'examen des données relatives aux révisions montre que l'activité des analystes est modérée. Pour un mois de décembre donné, on observe 400 à 1100 révisions (tableau 1 , révisions à la hausse et à la baisse), ce qui correspond à la réévaluation de près de $25 \%$ des prévisions. En d'autres termes, les analystes révisent leurs prévisions environ 4 fois par an. Nos contacts avec les firmes de courtage confirment cette pratique. Les révisions se produisent souvent lors de la publication des rapports annuels ou des états financiers trimestriels. Les changements dans la prévision moyenne et dans la dispersion des prévisions seront donc généralement de faible amplitude. La suite de l'analyse montre d'ailleurs une proportion élevée de révisions nulles. 
- Origine et traitement des données ${ }^{4}$ : les prévisions sont élaborées par les analystes des firmes de courtage, dont les services de recherche collectent et transfèrent les analyses. Chaque firme de courtage ne transmet qu'une prévision (ou une analyse) par titre. Pour un titre et un mois donné, le nombre de prévisions représente donc le nombre d'analystes et le nombre de firmes de courtage. Le tableau 2 illustre la provenance des prévisions portant sur les entreprises canadiennes, pour le mois de juin 1993, dernière période disponible sur la version détaillée de la base de données. 21 firmes de courtage canadiennes sont responsables de $86 \%$ des prévisions de bénéfices des entreprises canadiennes (2369 sur $2746 P A F$ ). Ces prévisions sont élaborées par 198 analystes financiers canadiens différents. S'ajoutent à cet effectif 191 analystes employés par 90 courtiers américains. Ceux-ci ne suivent généralement qu'un nombre réduit de titres et sont responsables de moins de $14 \%$ des prévisions disponibles. En moyenne, chaque analyste canadien produit des prévisions relatives à une douzaine de titres différents. Il est impossible d'identifier et donc de localiser ces courtiers. Nos contacts directs avec des firmes de courtage canadiennes révèlent qu'ils sont concentrés dans trois centres, soit Toronto, Montréal et Vancouver. Le tableau ci-dessous permet de comparer les situations canadienne et américaine. Les courtiers canadiens emploient en moyenne moins d'analystes participant à $I / B / E / S(9.4$ contre 18.8) et fournissent en moyenne la moitié du nombre de prévisions produites par leurs homologues américains. Il s'agit vraisemblablement d'un effet des différences de taille entre les courtiers des deux pays. Sous les autres dimensions, les contributions à $I / B / E / S$ sont similaires, qu'il s'agisse du rapport entre le nombre de titres suivis et celui des analystes ( 2 au Canada, 1.7 aux États-Unis) ou du nombre de prévisions par analyste qui s'élève à 12 aux Canada contre 11 aux États-Unis. Il ne semble pas exister de différences importantes entre les productions des prévisions dans les deux pays, si ce n'est la concentration plus importante des analystes et des prévisions dans des firmes de courtage de plus grande taille.

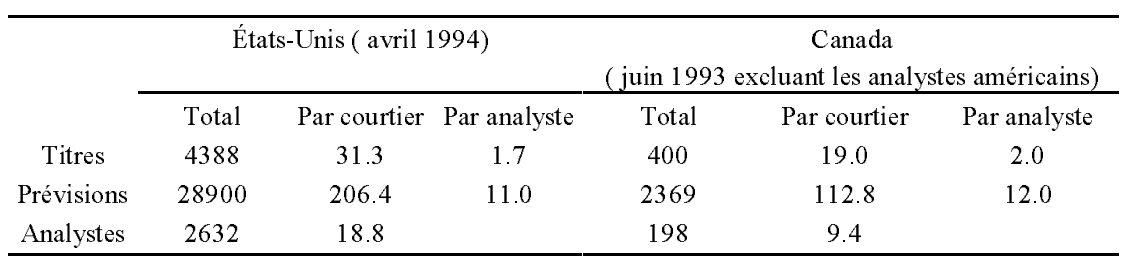

${ }^{4}$ Cette section a été élaborée à partir de la base de données détaillée de $I / B / E / S$. Elle a été réalisée avec 1'aide de Mesdames Jo-Ann Larsen, Jeanne Shea et Florence Eng de $I / B / E / S$ que nous tenons à remercier. Nous avons également eu recours aux responsables de recherche de firmes de courtage montréalaises. 
Les $P A F$ sont reçues sous des formes diverses, qui vont de rapports sur des compagnies individuelles à des sommaires portant sur l'ensemble des titres suivis par la firme participante. Les entreprises participantes fournissent les prévisions sur une base volontaire, mais dans certains cas, les prévisions sont sollicitées par $I / B / E / S$. En effet, chaque jour, les principales sources d'informations relatives aux entreprises sont examinées, (journaux financiers, bulletins électroniques) de façon à repérer les événements susceptibles d'influencer fortement les bénéfices des entreprises suivies. Lorsque de tels événements apparaissent, les analystes qui suivent le titre en question sont immédiatement sollicités pour réviser leurs prévisions. Divers outils de communication sont utilisés : transmission électronique, fax (60\%-70\%), téléphone, courrier. Les délais d'incorporation de l'information vont de moins de 4 heures, pour l'information reçue par fax, à moins de 24 heures pour les autres modes de communication. Toutefois, il semble courant que les firmes de courtage transmettent leur information sur une base mensuelle. Cette information est donc utilisée et diffusée avant même qu'elle ne soit transmise à $I / B / E / S$.

L'entrée des données se fait quotidiennement, dès réception des informations. Deux niveaux de vérification permettent ensuite d'assurer la cohérence transversale et temporelle des prévisions. Chacune des prévisions est vérifiée, dès le jour de sa réception de façon à s'assurer 1) que la base de calcul du BPA est cohérente au plan de la prise en compte des facteurs de dilution 2) que les ajustements relatifs aux fractionnements ou dividendes en actions ont été correctement effectués, 3) que les postes non-récurrents ont été traités correctement et 4) qu'il n'existe pas d'erreur dans l'identification du titre ou de l'analyste. Après ces vérifications, les $P A F$ sont incorporées à la base de données, qui subit continuellement un second niveau de vérification. Les distributions de prévisions sont analysées sur une base hebdomadaire, de façon à repérer les données extrêmes ainsi que les variations les plus importantes des prévisions moyennes et des dispersions. Ces situations particulières sont étudiées par les spécialistes de $I / B / E / S$, qui communiquent avec les analystes responsables des 75 à 100 estimés qui apparaissent les plus incohérents. Ce processus provoque le plus souvent une révision de la prévision en cause.

Cette analyse sommaire du processus de collecte et de vérification des prévisions apporte plusieurs éléments qui concernent l'aspect empirique du travail. En premier lieu, les prévisions proviennent d'un ensemble vaste d'analystes, et tous les grands courtiers canadiens contribuent à la collecte de prévisions... La quantité d'information

privée disponible aux investisseurs et indépendante de ces sources est 
vraisemblablement limitée. ${ }^{5}$ En second lieu, les prévisions portent sur des $B P A$ exprimés de façon cohérente, que ce soit en termes de facteur de dilution ou de traitement des postes extraordinaires. Le risque d'erreur semble par ailleurs minimisé par les diverses procédures mises en place par $I / B / E / S$ pour valider les données. Les variations importantes des distributions qui nous intéressent sont donc vraisemblablement imputables à des changements d'anticipations et non à des modifications de la définition des bénéfices. Troisièmement, la procédure de détection et de correction des prévisions extrêmes a vraisemblablement pour effet de réduire la dispersion de la prévision et de limiter du même coup les changements de cette dispersion. ${ }^{6}$ Finalement, la rapidité de la vérification et du traitement de l'information ainsi que la recherche des événements importants influençant les entreprises permettent d'anticiper une incorporation rapide de l'information dans les prévisions. Toutefois, la mesure des délais d'incorporation dépasse le cadre de cette étude, dont l'aspect descriptif sera limité aux caractéristiques temporelles des prévisions et aux relations entre les deux principales variables, soit les révisions et les changements dans la dispersion.

\section{3 Échantillon et mesure des variables}

Sur les 52330 PAF disponibles dans la base de données de départ, nous n'avons retenu que celles formulées entre janvier 1985 et mai 1994 (113 mois). Les titres qui intéressent moins de 6 analystes financiers ont été omis, puisque les mesures de dispersion ne peuvent être calculées avec précision pour ces observations. Les changements dans la distribution des anticipations des investisseurs ont été mesurés par les changements dans la distribution des prévisions de $B P A$ portant sur l'exercice en cours. Par conséquent, sur 12 prévisions portant sur le même exercice, il ne reste que 11 changements dans les $P A F$. L'échantillon comporte alors 19885 observations. Les mesures des changements dans l'anticipation moyenne $(A M)$ et dans la dispersion $(D I S P)$ des anticipations des agents sont notées $\Delta^{*} A M_{j, t}$ et $\Delta^{*} D I S P_{j, t}$ et calculées comme suit :

\footnotetext{
${ }^{5} \mathrm{Si}$ l'on élimine les 21 plus importantes firmes de courtage canadiennes, il semble subsister peu de sources d'information indépendantes, si ce n'est les services privés d'analyse de gestionnaires de fonds (qui utilisent eux-mêmes les analyses des firmes de courtage) et les entreprises elles-mêmes. Toutefois, les analystes financiers accèdent également à l'information des entreprises et cette source ne peut être considérée comme totalement privée au sens de ALV.

${ }^{6}$ I1 est donc vraisemblable, comme le soulignent Atiase et Bamber (1994, p.316), que la dispersion des analystes sous-estime généralement celle d'un ensemble plus vaste et plus hétérogène d'investisseurs diversement informés, dont les prévisions extrêmes ne sont pas filtrées.
} 


$$
\begin{gathered}
\Delta^{*} A M_{j, t} \equiv \frac{\Delta P M_{j, t}}{P_{j, t-1}}=\frac{\left(P M_{j, t}-P M_{j, t-1}\right)}{P_{j, t-1}} \\
\Delta^{*} D I S P_{j, t} \equiv \frac{\Delta E T_{j, t}}{P_{j, t-1}}=\frac{\left(E T_{j, t}-E T_{j, t-1}\right)}{P_{j, t-1}}
\end{gathered}
$$

où,

$P M_{j, t}$ : prévision moyenne du $B P A$ de l'entreprise j pour l'exercice financier en cours. L'indice t correspond au nombre de mois courus de l'exercice?.

$\dot{E} T_{j, t}: \quad$ écart type des prévisions de $B P A$ de l'entreprise j, publiées au mois $\mathrm{t}$ (l'écart type a été préféré au coefficient de variation afin que la variation dans la dispersion soit indépendante de la révision).

$\triangle^{*} A M_{j, t}$ : révision mensuelle de la prévision moyenne de $B P A$ de l'entreprise $\mathrm{j}$, normalisée par le prix du titre à la fermeture du mois précédent, noté $P_{j, t-1 \cdot 1}$.

$\Delta^{*} D I S P_{j, t}$ : variation mensuelle de l'écart type des prévisions de BPA de l'entreprise $\mathrm{j}$, normalisée par le prix du titre à la fermeture du mois précédent, noté $P_{j, t-1}$.

Les variables de contrôle qui découlent de l'étude de ALV sont le nombre d'analystes financiers suivant les titres et la dispersion des $P A F$ de début de période. La distribution du nombre d'analystes est tronquée et fortement asymétrique. Nous avons donc employé une variable binaire, en divisant l'échantillon suivant que les titres sont ou non suivis par moins de 12 analystes ${ }^{8}$.La dispersion de début de période est mesurée, comme dans le cas de Atiase et Bamber (1994), par le coefficient de variation des prévisions en début de période $(\mathrm{CV})$.

${ }^{7} \mathrm{t}=3$ peut donc correspondre à une prévision faite en mars pour un exercice finissant en décembre. Il peut également correspondre à une révision faite en décembre pour un exercice finissant en septembre.

${ }^{8}$ La valeur 12 divise approximativement l'échantillon en deux parties égales. Les spécifications alternatives basées sur le nombre d'analystes ou son logarithme donnent des résultats peu significatifs et instables, qui traduisent la forte non-linéarité des relations. 


$$
C V_{j, t-1}=\frac{\dot{E} T_{j, t-1}}{\left|P M_{j, t-1}\right|}
$$

Pour chacune des 19885 observations de l'échantillon $I / B / E / S$, une correspondance a été établie avec le fichier de rendements journaliers de TSE/Western. Les rendements mensuels et hebdomadaires des titres ont été calculés. L'équation (6) suggère une mesure du rendement total des titres. Cette mesure correspond parfaitement au modèle présenté dans la première section qui a été développé dans un cadre à deux actifs. Dans un tel cadre, les effets de diversification entre les actifs risqués n' apparaissent pas. Cependant, il est nécessaire de prendre en compte le risque systématique de ces actifs lorsque l'on teste empiriquement le modèle. Pour cela, nous avons mesuré le rendement résiduel des titres canadiens en éliminant les effets conjoints du risque systématique du titre et du rendement de marché. Ce rendement a été calculé en utilisant plusieurs modèles de génération des rendements (CAPM, modèle de marché et modèle de rendements ajustés pour le marché). ${ }^{9}$ Le taux de rendement de l'actif sans risque est mesuré par celui des bons du Trésor, relevé dans la Revue de la Banque du Canada. L'indice de marché utilisé est le TSE 300 ajusté pour le rendement en dividendes.

\section{4 Évolution du consensus et de la dispersion}

Le tableau 3 montre l'évolution des moyennes et médianes des distributions de $P M_{j, t}$, $\Delta^{*} A M_{j, t}, E T_{j, t}$ et $\Delta^{*} D I S P_{j, t}$ au long de l'exercice financier, pour l'ensemble de la période. La prévision moyenne, $P M_{j, t}$, augmente légèrement au cours des 4 premiers mois de l'exercice, pour diminuer ensuite et revenir, à la fin de l'exercice financier, au niveau qui prévalait au premier mois de l'exercice financier. Les moyennes et médianes des distributions des révisions, $\Delta^{*} A M_{j, t}$, sont en moyenne positives au cours des mois 2 et 3 , alors que les révisions suivantes sont en moyenne négatives et de plus en plus importantes en valeur absolue. Par ailleurs, on observe la même tendance pour la médiane des révisions, mais cette dernière est toujours inférieure en valeur absolue à la moyenne. La distribution des prévisions moyennes et des révisions est donc légèrement asymétrique. Cette évolution concorde avec les résultats de Brown,

\footnotetext{
${ }^{9}$ Le détail du calcul des rendements résiduels se trouve à la figure 2.
} 
Richardson et Trzcinka (1991) qui montrent qu'en début d'année, les analystes afficheraient généralement plus d'optimisme quant aux $B P A$ futurs. Le $B P A$ de l'année précédente serait employé initialement comme prévision pour l'exercice suivant. Cette prévision initiale serait révisée, généralement de façon optimiste, au cours des premiers mois de l'exercice. Par la suite, on retrouverait un processus de révisions à la baisse. Enfin, on observe une importante proportion de révisions nulles, qui se situe en moyenne à $17.44 \%$.

Les distributions des écarts types, ainsi que des changements dans la dispersion, $E_{j, t}$ et $\Delta^{*} D I S P_{j, t}$ indiquent une évolution similaire à celle des prévisions. Alors qu'aux États-Unis, on observe une réduction monotone de la dispersion tout au long de l'exercice (Brown, Foster et Noreen, 1985), au Canada, cette dispersion augmente jusqu' au quatrième mois. La dispersion des anticipations ne diminue qu'à partir du cinquième mois et, en fin d'exercice, elle est à peu près similaire à celle qui prévalait en début d'exercice. Il est probable que plusieurs analystes se contentent de reporter leur prévision de l'exercice précédent à l'exercice financier suivant, jusqu'au moment où le rapport annuel est publié. La dispersion des prévisions en début d'exercice est vraisemblablement une mesure peu fiable de l'hétérogénéité des anticipations des investisseurs. Ici encore, il existe une proportion importante de changements nuls dans la dispersion, soit $25.87 \%$. La proportion de changements nuls dans la dispersion dépasse celle observée dans les révisions. Des révisions peuvent donc se produire sans modifier la dispersion, ce qui provient du fait que l'écart type rapporté par $I / B / E / S$ est tronqué à la troisième décimale.

Les 2 variables $\Delta^{*} A M_{j, t}$ et $\Delta^{*} D I S P_{j, t}$ ne sont pas totalement indépendantes. La figure 1 présente, dans le plan $\Delta^{*} A M_{j, t}$ et $\Delta^{*} D I S P_{j, t}$ les courbes correspondant aux fractiles de la distribution des révisions, pour chacun des déciles de la distribution des révisions. Les changements dans la dispersion sont en moyenne plus importants (en valeur absolue) dans les fractiles extrêmes de la distribution des révisions. Ce résultat est cohérent avec les conclusions de Holthausen et Verrecchia (1990). Toutefois, pour des révisions importantes du consensus à la hausse ou à la baisse, le changement dans la dispersion peut être de même sens ou de sens opposé. Le coefficient de corrélation entre les variables $\Delta^{*} A M$ et $\Delta^{*} D I S P$ n'est donc que de .0592 (voir tableau 3 ). Les relations entre $\Delta^{*} A M$ et $\Delta^{*} D I S P$ et les variables de contrôle $N B A$ (nombre d'analytes) et $C V$ ne posent pas de problèmes aigus de collinéarité. La section 2 a permis de discuter les problèmes que soulève l'estimation des anticipations des agents par les $P A F$, de proposer des mesures pour les changements dans la distribution des 
anticipations des agents et de présenter sommairement les données. La section suivante est consacrée à l'analyse formelle de la relation établie en (6).

3

\section{MÉTHODOLOGIE ET RÉSULTATS}

L'analyse de la relation entre les rendements des titres et les changements dans la prévision moyenne et la dispersion des $P A F$ est menée de deux manières différentes. En premier lieu, nous testons les hypothèses selon lesquelles les coefficients de l'équation (6), $\boldsymbol{\alpha}_{1}$ et $\boldsymbol{\alpha}_{2}$ sont respectivement positif et négatif en utilisant une étude de type associatif, basée sur un modèle de régression. Cette première partie permet d'estimer les paramètres $\boldsymbol{\alpha}_{\mathrm{i}}$, mais ne donne aucune information sur l'évolution des rendements autour des changements importants dans le consensus et la dispersion. Elle est donc complétée par une analyse résiduelle présentant l'évolution des rendements de portefeuilles formés sur la base des changements dans la distribution des $P A F$.

\subsection{Analyse associative}

-Méthodologie et hypothèses : dans cette section, les coefficients moyens mensuels $\left(\alpha_{i}, \mathrm{i}=1,2\right)$ associés aux changements (normalisés par le prix) de la moyenne et de l'écart type des $P A F$ sont estimés à l'aide d'une procédure en 2 étapes ${ }^{10}$. Dans une première étape, pour chacun des 113 mois considérés, les paramètres $\alpha_{\mathrm{it}}$ de l'équation ci-dessous sont estimés :

$$
R_{j t}=\alpha_{0 t}+\alpha_{1 t} \Delta^{*} A M_{j t}+\alpha_{2 t} \Delta^{*} D I S P_{j t}+\varepsilon_{j t}
$$

Dans une seconde étape, les coefficients associés aux changements dans l'anticipation moyenne $\left(\Delta^{*} A M_{j, t}\right)$ et dans la dispersion des PAF $\left(\Delta^{*} D I S P_{j, t}\right)$ sont estimés en calculant la moyenne équipondérée des coefficients $\boldsymbol{\alpha}_{\text {it }}($ Fama et French, 1992) et un test t permet de juger de leur niveau de signification ${ }^{11}$.

\footnotetext{
${ }^{10}$ I1 existe deux autres méthodes d'estimation utilisables. La première consiste à calculer une moyenne pondérée des coefficients, de façon à tenir compte de la précision avec laquelle les coefficients sont estimés (Huang et Litzenberger, 1988). Cette méthode a été utilisée pour confirmer les résultats présentés ici. La seconde méthode consiste à regrouper les observations mensuelles pour estimer les coefficients moyens (pooling). Cette méthode n'est pas utilisée ici puisqu'elle suppose la stabilité intertemporelle des coefficients, qui n'est vraisemblablement pas respectée.

${ }^{11}$ Le détail des calculs des coefficients moyens et des tests correspondants est présenté au tableau 4.
} 
Les travaux portant sur les relations entre les rendements des titres et les révisions de la prévision moyenne diffusée par $I / B / E / S$ (Brown, Foster et Noreen, 1985 ; Dimson et March, 1984) ayant mis en évidence le décalage qui existe entre les révisions des $P A F$ et la publication de ces révisions par $I / B / E / S$, nous avons mesuré le rendement résiduel à 3 dates différentes : le mois précédant, le mois correspondant et le mois suivant les changements dans la moyenne et la dispersion des prévisions. Ils sont respectivement notés Res_1, Res0 et Res1.

- Résultats : les résultats sont présentés au volet $\mathrm{A}$ du tableau $4^{12}$. En moyenne, le coefficient $\alpha_{1}$ est de 0.97 au temps $-1,0.68$ au temps 0 et 0.42 au temps +1 . Ces coefficients sont statistiquement significatifs au seuil de 1 pour 1000. L'effet maximal est observé au mois précédant l'annonce, ce qui est compatible avec les résultats antérieurs et traduit le fait que les prévisions des analystes et l'information sousjacente à ces prévisions atteignent le marché bien avant leur diffusion par $I / B / E / S$. L'effet des révisions est perceptible au cours des trois mois étudiés, bien que l'ampleur du coefficient décline. Ce phénomène a deux origines. En premier lieu, le marché semble incorporer lentement la totalité des implications des nouvelles informations. Ce post announcement drift qui a été, entre autres, analysé par Bhushan (1994) semble lié aux frais de transaction qui limitent l'ajustement des cours. En second lieu, les révisions des prévisions moyennes des analystes sont corrélées sériellement. En effet, Brown, Foster et Noreen (1985), ainsi que L'Her et Suret (1991) ont déjà mis en évidence ce problème de récurrence des révisions moyennes, $\Delta^{*} A M_{j, r}$. Ce problème de récurrence a été traité, en répliquant l'analyse après avoir éliminé de l'échantillon toutes les observations précédées d'une révision de même nature. Cette modification a pour effet de réduire l'ampleur des coefficients, mais ne résout pas le problème de diminution importante du coefficient $\alpha_{1}$ du mois -1 au mois 1. Les résultats concernant ce traitement ne sont pas reportés ici, car l'analyse associative est mal adaptée pour résoudre ce problème. C'est la raison pour laquelle nous utilisons dans la section 3.2 une analyse résiduelle plus propice à rendre compte du comportement des prix des titres autour des changements dans les distributions des $P A F$.

L'ampleur des coefficients est conforme aux attentes. En fait, à partir de l'équation A23, il est possible d'appréhender l'effet d'une variation de l'anticipation moyenne

\footnotetext{
${ }^{12} \mathrm{~L}$ 'analyse associative étant sensible aux valeurs extrêmes, nous avons limité l'étendue des distributions en ramenant les observations supérieures au $99^{e}$ centile ou inférieures au $1^{\text {er }}$ centile à la valeur de ces centiles.
} 
(normalisée) des investisseurs informés sur le rendement de l'actif risqué de l'ordre de .9 :

$$
\frac{\partial R}{\partial \Delta^{*} A M}=\frac{1}{R}=\frac{1}{1+r_{f}} \approx .9 \operatorname{sir}_{f}=.10
$$

Deux arguments contraires laissent toutefois penser que le coefficient observé $\alpha_{1}$ diffère de cette valeur théorique attendue. Tout d'abord, la valeur de 9 est dérivée dans le cadre d'un modèle monopériodique où les annonces de révision n'ont aucun effet sur les anticipations de bénéfice des périodes futures. La persistance est donc absente de la modélisation. Or, en pratique, la persistance est vraisemblablement supérieure à 0 et l'ajustement des cours à la révision des prévisions de bénéfice pourrait donc excéder l'unité. Par ailleurs, si les vérifications empiriques sont basées sur des rendements résiduels, c'est-à-dire que les effets conjoints du risque systématique et du marché sont éliminés du calcul du rendement des titres, ce n'est pas le cas pour les révisions. Cette différence dans le traitement des variables expliquée et explicative peut alors expliquer un coefficient $\alpha_{1}$ observé inférieur à l'unité. L'ampleur de ce coefficient dépendra donc de ces 2 effets contraires. Les coefficients se situent entre 1.4 (lorsqu'on limite l'échantillon aux titres largement suivis) et un minimum de .78 . En particulier, le coefficient de .97 observé au temps -1 pour l'ensemble de l'échantillon indique qu'une révision qui représente une proportion donnée du prix se traduit par un rendement excédentaire à peu près identique.

Les coefficients $\alpha_{2}$ sont négatifs et statistiquement significatifs. Ils sont respectivement de $-0.37,-.24$ et -0.37 pour Res_1, Res0 et Res1. L'ampleur de ce coefficient est plus difficile à anticiper. En effet, s'il est possible de déterminer analytiquement l'impact d'une variation de la dispersion des anticipations (normalisée) des investisseurs informés sur le rendement de l'actif risqué (équation (6)),

$$
\frac{\partial R}{\partial \Delta^{*} D I S P}=-\frac{\sigma_{e} \bar{S}}{r R}
$$

il est toutefois difficile d'avancer des valeurs de l'inverse de la précision du signal reçu par les investisseurs informés $\left(\sigma_{e}\right)$, de la tolérance au risque agrégée des investisseurs (r) et de l'offre nette d'actif risqué per capita, $\bar{S}$. Nous retiendrons seulement que l'effet des changements dans la dispersion des $P A F$ est stable pour les 
3 mois analysés. En effet, la corrélation sérielle des changements dans la dispersion est négligeable. Par ailleurs, cet effet est significatif, mais inférieur en valeur absolue à celui des changements dans le consensus.

Il existerait donc, au Canada, une relation positive entre la révision des prévisions moyennes des analystes financiers et le rendement résiduel des titres et une relation négative entre le changement dans la dispersion et le rendement résiduel des titres. Le $\mathrm{R}^{2}$ moyen calculé est toutefois faible, il est de l'ordre de $5 \%$ pour le résidu au temps -1 et de $2.8 \%$ au temps +1 .

- Spécifications complémentaires : la sensibilité des résultats présentés au tableau 4 a été étudiée par rapport à la méthodologie, au choix du modèle générateur des rendements, et à l'introduction des variables de contrôle. Dans un premier temps, nous avons vérifié que les coefficients et les niveaux de signification n'étaient pas sensibles à la méthode d'estimation. Le volet $\mathrm{B}$ du tableau 4 montre que l'utilisation de la méthode de Huang et Litzenberger (1988) conduit à des estimations identiques. Les calculs ont ensuite été répliqués en substituant le modèle de rendements ajustés pour le marché au CAPM, ce qui permet de disposer de 19885 observations au lieu de 16442. Les coefficients et les niveaux de signification, rapportés au volet $\mathrm{C}$ du tableau 4 , sont du même ordre que ceux obtenus précédemment.

Dans une deuxième étape, les régressions ont été menées en divisant l'échantillon sur la base du nombre d'analystes suivant les titres et en introduisant le coefficient de variation comme mesure de la dispersion en début de période. Les résultats apparaissent aux volets $\mathrm{D}$ et $\mathrm{E}$ du tableau 4. Le coefficient de variation ne joue aucun rôle dans la relation. La division en deux groupes sur la base du nombre d'analystes ne remet pas en cause les relations observées pour l'ensemble de l'échantillon. Toutefois, la réaction des prix aux révisions est plus importante lorsque les titres sont largement suivis. En effet, le coefficient du résidu observé au temps -1 est de 1.40 contre .91 dans le second groupe. Il est possible que les changements de l'anticipation moyenne des investisseurs soient mieux estimés par les changements de prévisions des analystes lorsque ceux-ci sont nombreux. Les coefficients associés à la variable $\Delta^{*} D I S P$ sont généralement négatifs, sauf pour le groupe des titres largement suivis, au temps 0 . Il est, dans ce cas, non significatif. Seulement 3 des coefficients (sur 6) sont statistiquement significatifs au seuil de $5 \%$. Deux autres ne sont significatifs qu' au seuil de $12 \%$. Ce résultat a deux explications. La division de l'échantillon réduit sensiblement la puissance des tests puisque l'on estime ici des coefficients mensuellement. Ceux-ci sont estimés avec une variance élevée, ce qui influence les 
valeurs t. En second lieu, il semble clair que le nombre d'analystes modifie la façon dont les changements dans la dispersion des anticipations des agents est liée aux rendements. Toutefois, il s' agit là d'une relation complexe dont l'étude dépasse le cadre de ce travail.

L'approche associative nous permet d'estimer les coefficients moyens de la relation entre le rendement des titres et les changements dans la distribution des anticipations des agents, mais ne nous renseigne pas sur le comportement des prix des titres autour de ces changements. Les résultats des études empiriques antérieures portant sur les $P A F$ font pourtant clairement ressortir qu'il existe un décalage temporel important entre le moment où les révisions des $P A F$ sont faites et le moment où les révisions des anticipations sont publiées par $I / B / E / S$. Afin d'étudier le comportement des rendements des titres autour des révisions importantes des $P A F$, une analyse résiduelle des rendements de portefeuilles de titres construits sur la base des changements dans la distribution des $P A F$ est utilisée pour compléter les résultats de la section précédente.

\subsection{Analyse résiduelle}

- Formation des portefeuilles et hypothèses : pour chacun des 113 mois considérés, nous avons effectué un premier tri descendant sur la base de la révision pour former trois tiers : le premier (dernier) tiers correspondant aux révisions les plus optimistes (pessimistes). Par la suite, pour chacun de ces tiers, nous avons effectué un second tri ascendant sur la base des changements dans la dispersion. Les 3 portefeuilles formés dans la première étape ont été à nouveau subdivisés en 3 de sorte que nous avons pour chaque mois 9 portefeuilles. Le premier correspond aux révisions les plus favorables et aux plus grandes réductions de la dispersion des anticipations. Le neuvième portefeuille correspond aux révisions les plus défavorables et aux plus grandes augmentations de la dispersion des anticipations. Les portefeuilles 1, 3, 7 et 9 seront notés de la façon suivante :

$\mathrm{P}^{+-}$: révisions à la hausse, avec diminution de la dispersion (portefeuille 1). $\mathrm{P}^{++}$: révisions à la hausse, avec augmentation de la dispersion (portefeuille 3 ). $\mathrm{P}^{--}$: révisions à la baisse, avec diminution de la dispersion (portefeuille 7). $\mathrm{P}^{-+}$: révisions à la baisse, avec augmentation de la dispersion (portefeuille 9).

Les 4 portefeuilles comptent environ 2200 observations. Si la relation (6) tient, on devrait observer la relation suivante entre la performance de ces quatre portefeuilles, 
notée perf ', où le premier exposant représente le sens de la révision et le second le changement dans la dispersion : perf $^{+-}>$perf $^{++}>0>\operatorname{perf}^{--}>$perf ${ }^{-+}$.

- Méthodologie : 1'analyse résiduelle repose sur la comparaison des rendements des 4 portefeuilles $\mathrm{P}^{+-}, \mathrm{P}^{++}$,

$\mathrm{P}^{--}$et $\mathrm{P}^{-+}$calculés sur une base hebdomadaire. La semaine 0 a été fixée à la première semaine du mois au cours duquel les prévisions $I / B / E / S$ sont publiées. Le résidu moyen $\left(\mathrm{RM}_{\mathrm{t}}\right)$ et le résidu moyen cumulé sur la période allant de 0 à $\mathrm{t}\left(\mathrm{RMC}_{0}^{\mathrm{t}}\right)$ sont calculés de la façon suivante : $\mathrm{RM}_{\mathrm{t}}=(1 / \mathrm{N}) \sum_{\mathrm{i}=1}^{\mathrm{N}} \mathrm{e}_{\mathrm{it}}, \mathrm{t}=-26, \ldots,+26$, et $\mathrm{RMC}_{0}^{\mathrm{t}}=$ $\sum_{\mathrm{s}=0}^{\mathrm{t}} \mathrm{RM}_{\mathrm{ots}}$ où $\mathrm{N}$ représente le nombre de titres compris dans le portefeuille et $\mathrm{e}_{\mathrm{it}}$ représente le rendement résiduel du titre i au temps $t\left(e_{i t}=R_{i t}-E\left(R_{i t}\right)\right.$ où $E\left(R_{i t}\right)$ est mesuré à partir d'un modèle générateur des rendements). Le test $t$ utilisé pour déterminer si les $\mathrm{RM}_{\mathrm{t}}$ sont significativement différents de 0 est calculé en prenant l'écart type des RM sur la période $[-26,+26]$ comme estimateur de l'écart type des RM.

Les différences entre les RM et les RMC des deux portefeuilles $\mathrm{P}^{+}$. sont notées $\mathrm{DIF}^{+}$; $\mathrm{DIFC}^{+}$; celles qui séparent les rendements des deux portefeuilles $\mathrm{P}^{-}$- sont notées DIF ${ }^{-}$et DIFC ${ }^{-}$. Comme le suggèrent Ou et Penman (1989), DIF et DIFC peuvent être considérées comme les rendements d'un portefeuille dans lequel les titres (. +) ont été vendus à découvert pour acquérir les titres (. - ). Un test t est utilisé pour déterminer dans quelle mesure les différences hebdomadaires (DIF) et les différences cumulées (DIFC) diffèrent statistiquement de 0

$$
t_{D I F}=\frac{D I F_{t}}{\sigma_{D I F}} \text { et } t_{D I F C}=\frac{D I F C_{t}}{\sigma_{D I F} / \sqrt{n}}
$$

où $\sigma_{\mathrm{DF}}$ est l'écart type des 52 différences hebdomadaires mesurées entre les semaines -26 et +26 . Dans le cas des différences cumulées, l'écart type est divisé par la racine carrée du nombre de périodes de cumul. Il s'agit donc de tests conventionnels, qui prennent en compte la corrélation transversale positive potentielle entre les rendements anormaux des titres composant les portefeuilles (Chandra et Balanchandran, 1990). 
- Résultats : la figure 2 représente les $\mathrm{RMC}$ des quatre portefeuilles $\mathrm{P}^{+-}, \mathrm{P}^{++}, \mathrm{P}^{-}$ ${ }^{-}$et $\mathrm{P}^{-+}$pour la période de 52 semaines entourant la semaine $0[-26,+26]$. Les RMC des portefeuilles $\mathrm{P}^{+-}$et $\mathrm{P}^{++}$sont positifs. À l'inverse, les RMC des portefeuilles $\mathrm{P}-$ - et $\mathrm{P}^{-+}$sont négatifs. Les RMC des deux portefeuilles centraux ( les portefeuilles 4 et 6 composés de titres pour lesquels les révisions ont été faibles ou nulles) sont également illustrés. Ces résultats sont conformes à ceux attendus, c'est-à-dire qu'il existe une relation positive entre les révisions des $P A F$ et les rendements des titres. En raison du laps de temps qui s'écoule entre la production des $P A F$ et leur diffusion par $I / B / E / S$, les RMC des quatre portefeuilles sont significativement différents de zéro avant la semaine 0 . Par ailleurs, pour des révisions identiques en valeur absolue, l'effet des révisions négatives sur le prix des titres est plus prononcé que celui des révisions positives $\left(\mathrm{RMC}_{36}^{26}\right.$ sur du portefeuille $\mathrm{P}^{-+}=-18.23 \%$; $\mathrm{RMC}$ du portefeuille $\mathrm{P}^{+-}=+13.91 \%$ ), ce qui est du même ordre que les résultats des études antérieures (L'Her et Suret, 1991). Notons, que, même si l'objet de l'étude est essentiellement l'effet de la dispersion sur le prix des titres, une stratégie couverte qui consisterait à vendre à découvert les titres du portefeuille $\mathrm{P}^{-+}$et à acheter les titres du portefeuille $\mathrm{P}^{+-}$procurerait un $\mathrm{RMC}_{0}^{26}$ extrêmement important de 10.55 \%.

Les principales données relatives aux RM des 4 portefeuilles, pour les semaines - 13 à +13 apparaissent au tableau 5. Pour limiter la taille du tableau, DIF n'est pas reportée, mais le test portant sur DIF est présenté. $\mathrm{DIF}^{+}$, c'est-à-dire la différence entre les rendements des portefeuilles $\mathrm{P}^{+-}$et $\mathrm{P}^{++}$n'est significative pour aucune des semaines de -13 à +13 . Par contre, DIF', c'est-à-dire la différence entre les rendements des portefeuilles $\mathrm{P}^{--}$et $\mathrm{P}^{-+}$est significative les semaines -4 et -2 . Les $\mathrm{RMC}_{0}^{\mathrm{t}}$ des 4 portefeuilles sont également présentés dans la seconde partie du tableau 5 ( $t=0$ à 26). L'écart entre les RMC des deux portefeuilles composés de titres révisés à la baisse est plus important que l'écart observé entre les deux portefeuilles de titres révisés à la hausse. Il est, pour la semaine 26 , de 29 points de base dans le premier cas $\left(\mathrm{RMC}_{0}^{26}=5 \%\right.$ pour $\mathrm{P}^{+-}$et $\mathrm{RMC}_{0}^{26}=2.1 \%$ pour $\left.\mathrm{P}^{++}\right)$et de 41 points de base dans le second cas $\left(\mathrm{RMC}_{0}^{26}=-1.4 \%\right.$ pour $\mathrm{P}^{--}$et $\mathrm{RMC}_{0}^{26}=-5.5 \%$ pour $\left.\mathrm{P}^{-+}\right)$. $\mathrm{DIFC}^{+}$, c'est-à-dire la différence entre les RMC des portefeuilles révisés à la hausse n'est statistiquement différente de 0 au seuil de signification de $5 \%$ qu'à partir de la semaine 9 , alors que DIFC ${ }^{-}$est significative dès la semaine 1 . Cette asymétrie entre les $\mathrm{RMC}$ et les DIFC pour les révisions à la hausse et à la baisse a 2 origines possibles. L'autocorrélation positive des révisions est plus forte pour les révisions positives et réduit par conséquent l'effet de surprise, ce qui limite l'impact sur les prix. Les restrictions sur les ventes à découvert freinent les ajustements de prix pour les révisions à la baisse. 
La variation de la dispersion des $P A F$ a donc un effet important sur le rendement des titres. Un gestionnaire qui aurait construit des portefeuilles sur la base des changements dans la distribution des $P A F$ durant la période de janvier 1985 à mai 1994 aurait obtenu en moyenne des rendements annualisés de $5.88 \%\left(\mathrm{DIFC}^{+}\right)$et de $8.36 \%$ ( $\left.\mathrm{DIFC}^{-}\right)$. Notons que ce résultat est obtenu en considérant un très vaste ensemble de titres et que le rendement annualisé serait sans doute plus important si un gestionnaire ne considérait que les variations les plus importantes dans le consensus et la dispersion. Afin de s'assurer de la robustesse de ce résultat, nous avons donc mené une analyse de sensibilité à différents paramètres.

- Analyse de sensibilité : la sensibilité des résultats de l'analyse résiduelle à la composition de l'échantillon, au choix du modèle générateur des rendements et au choix des indices de marché a été examinée. Ces résultats sont présentés ci-dessous.

Effet janvier: de nombreuses études ont mis en évidence des RM positifs importants des titres à faible capitalisation boursière au cours du mois de janvier. L'analyse a donc été répétée en omettant ces observations. Les résultats obtenus, dont un sommaire est présenté au volet A du tableau 6 , n'indiquent aucune différence importante par rapport aux RM, RMC et tests statistiques obtenus à partir de l'échantillon complet.

Nombre d'analystes: afin de prendre en compte l'influence du nombre d'analystes financiers suivant le titre, l'échantillon total a été divisé en deux groupes d'effectifs identiques. ${ }^{13}$ Le premier rassemble les entreprises suivies par 6 à 11 analystes, alors que le second rassemble les titres suivis par 12 analystes et plus. Les RMC sont présentés aux figures 3 (moins de 12 analystes) et 4 (12 analystes et plus). Les résidus cumulés à partir du temps 0 ainsi que les tests statistiques aux semaines $0,6,12$ et 24 sont présentés pour chacun des groupes au volet A du tableau 6 .

Les deux figures diffèrent, mais les résultats obtenus pour l'ensemble des observations demeurent valables, sauf pour les révisions positives relatives aux titres largement suivis. Dans ce cas, les changements dans la dispersion ne semblent pas influencer les rendements. Il semble donc que le nombre d'analystes financiers influence la

\footnotetext{
${ }^{13}$ L'analyse résiduelle utilisée ici ne permet pas de tenir compte d'un nombre élevé de variables de contrôle, puisque chacune réduit la taille des portefeuilles de façon importante. Nous faisons donc l'hypothèse que la dispersion de début de période, qui constitue l'autre variable de contrôle, est distribuée aléatoirement dans les divers portefeuilles.
} 
relation entre les changements dans la distribution des prévisions et les rendements. Cette constatation est cohérente avec les résultats obtenus par la méthode associative. Elle ne remet pas en cause nos résultats, mais confirme les hypothèses avancées par ALV. Dans le cas des titres faiblement suivis, les variations dans la dispersion semblent fortement anticipées. La différence entre les RMC est significative dès la semaine -12. Ce résultat semble partiellement lié aux situations de récurrence, c'est à dire à la présence de révisions et de changements dans la dispersion de même nature au cours de mois successifs. Ce phénomène a dont été analysé.

Récurrence : une situation de récurrence survient lorsqu'un titre fait l'objet d'une révision importante ou d'un changement de dispersion plusieurs mois de suite. Il se trouve alors incorporé au même portefeuille durant plusieurs périodes consécutives, ce qui est à l'origine d'effets de décalage entre les changements dans la distribution des $P A F$. Pour limiter ce problème, les cas de récurrence portant sur un même exercice ont été omis. Pratiquement, lorsqu'un titre se trouve inclus dans un même portefeuille durant plusieurs mois consécutifs, seule la première occurrence est prise en compte. Les résultats ainsi obtenus, pour l'ensemble des observations, sont illustrés à la figure 5. Celle-ci se distingue de la figure 2 sous deux aspects. Les RMC sont généralement de moindre amplitude, que ce soit lors des révisions en hausse ou en baisse des BPA. En second lieu, l'effet des changements de dispersion sur les rendements intervient plus tardivement. Il n'est pas perceptible avant la semaine 4 lors des révisions en hausse et -3 lors des révisions en baisse. Les effets d'anticipations perceptibles dans les résultats présentés jusqu'ici sont donc, au moins en partie, imputables aux situations de récurrence. Toutefois, après 26 semaines, il existe une différence significative entre les RMC des portefeuilles suivant qu'ils sont composés de titres dont la dispersion a augmenté ou diminué.

Choix du modèle générateur des rendements : la valeur des rendements résiduels peut dépendre du modèle générateur des rendements choisi. Par ailleurs, le CAPM subit des remises en cause fréquentes. Nous avons donc utilisé deux modèles générateurs des rendements soit le modèle de rendements ajustés pour le marché (The Market Adjusted Returns Model) et le modèle de marché. Les résultats sommaires, présentés au volet $B$ du tableau 6 , montrent que les conclusions ne sont pas modifiées par le choix de ce modèle.

Choix des indices de marché : les calculs ont été répliqués à l'aide d'un indice pondéré selon la capitalisation boursière des entreprises composant notre échantillon, puis à l'aide d'un indice équipondéré incorporant l'ensemble des titres négociés à la 
bourse de Toronto. Les résultats obtenus sont identiques à ceux observés lorsque l'indice TSE est utilisé. Ceci indique que nos résultats ne sont pas imputables au caractère particulier des titres qui composent l'échantillon.

\section{CONCLUSION}

L'étude menée ici analyse, tant sur le plan théorique qu'empirique, les relations entre les variations des deux premiers moments de la distribution des anticipations des agents et le rendement des titres. Elle montre que, dans le cadre d'un modèle d'équilibre en attentes rationnelles bruité, il est possible d'établir une expression du prix des titres qui est une fonction linéaire positive du consensus et une fonction linéaire négative de la dispersion des anticipations. La transformation de cette relation permet de mettre en évidence une forme empirique testable entre le rendement réalisé des titres et les changements dans les deux premiers moments de la distribution des anticipations normalisés par le prix des titres. Les anticipations des agents sont mesurées par les prévisions des analystes financiers distribuées par $I / B / E / S$ (Institutional Brokers Estimate System). A partir des prévisions détaillées réalisées depuis 1974, l'étude dresse un tableau de l'industrie de l'analyse financière canadienne. 21 firmes de courtage canadiennes et 50 firmes américaines fournissent respectivement $90 \%$ et $10 \%$ des prévisions portant sur les entreprises canadiennes. La couverture des titres canadiens ne diffère pas sensiblement de celle observée aux États-Unis, à la différence près que les firmes de courtage canadiennes semblent de plus petite taille que leurs homologues américaines.

Les résultats de l'étude empirique se fondent sur un échantillon de 19885 prévisions réalisées de janvier 1985 à mai 1994 par plus de 6 analystes financiers. Les principaux résultats sont les suivants. En premier lieu, conformément à la proposition de Holthausen et Verrecchia (1990), les changements les plus importants de la dispersion des anticipations des agents coïncident avec les révisions du consensus les plus importantes en valeur absolue. Toutefois, le signe de la variation de la dispersion est indépendant du signe de la révision du consensus. Les variations du consensus et de la dispersion sont concomitantes, ce qui peut expliquer que les travaux antérieurs, qui ont généralement étudié la variation dans la dispersion séparément, n'aient pas réussi à isoler l'effet de la dispersion sur le prix des titres risqués. Par ailleurs, les résultats de l'étude associative indiquent que l'effet d'une variation mensuelle du consensus sur le rendement mensuel contemporain des titres est plus important que l'effet d'une variation de la dispersion. Les coefficients associés aux variations du consensus et de 
1a dispersion des anticipations sont peu sensibles à l'introduction des variables de contrôle suggérées par Abarbanel, Lanen et Verrecchia (1994) : le nombre d'analystes financiers suivant les titres et la dispersion des anticipations mesurée en début de période. Les résultats de l'analyse résiduelle montrent que si l'on contrôle pour le sens de la révision du consensus, la différence entre les résidus moyens cumulés de portefeuilles pour lesquels la dispersion a diminué ou augmenté est significative, surtout pour les révisions du consensus à la baisse. Les résultats de l'analyse résiduelle sont peu sensibles à la composition de l'échantillon (effet janvier, nombre d'analystes, récurrence des révisions), au choix du modèle générateur des rendements et au choix de l'indice de marché utilisés lors de l'élaboration des tests.

Ces résultats peuvent vraisemblablement être invoqués pour expliquer des anomalies persistantes qui contredisent les modèles conventionnels. Les effets de taille et d'information incomplète (Merton, 1987) peuvent simplement être liés au fait que les anticipations des agents sont plus hétérogènes. Par ailleurs, les résultats semblent importants pour les recherches qui tentent d'expliquer la réaction des prix des titres à l'arrivée d'information comptable. En effet, l'information influence simultanément le consensus et la dispersion des anticipations et tous deux peuvent en conséquence modifier les prix. Tout modèle qui néglige ce double effet est vraisemblablement incomplet. Les travaux ultérieurs devront sans doute examiner les effets des variations dans les autres moments de la distribution des anticipations ou les effets des variations des 2 premiers moments des révisions des prévisions portant sur des horizons plus éloignés. 


\section{BIBLIOGRAPHIE}

Abarbanel, J.S., W.N. Lanen et R.E. Verrecchia, Analysts' Forecasts as Proxies for Investors Beliefs in Empirical Research, Working Paper, University of Michigan (janvier 1994).

Ajinkya, B.B., R. Atiase et J. Gift, "Volume of Trading and the Dispersion in Financial Analysts' Earnings Forecasts ", The Accounting Review (avril 1991) : 389-401.

Amihud, Y. et H. Mendelson, "The Effects of Beta, Bid-Ask Spread, Residual Risk, and Size on Stock Returns ", The Journal of Finance (juin 1989) : 479-486.

Arbel, A. et P. Strebel, "The Neglected and Small Firm Effects ", The Financial Review (novembre 1982) : 201-218.

Atiase, R.K. et L.S. Bamber, "Trading Volume Reaction to Annual Accounting Earnings Announcements ", Journal of Accounting and Economics 17 (1994) : 309-329.

Barry, C. B. et R.H. Jennings, "Information and Diversity of Analyst Opinion ", Journal of Financial and Quantitative Analysis (juin 1992) : 169-183.

Barry, C. et S. Brown, "Differential Information and the Security Market Equilibrium ", The Journal of financial and Quantitaitve Analysis (décembre 1985) : 407-422.

Bhushan, R., " An Informational Efficiency Perspective on the Post-Announcement Drift ", Journal of Accounting and Economics 18 (1994) : 45-65.

Brown, L.D., G.D. Richardson et C.A. Trzcinka, " Strong-Form Efficiency on the Toronto Stock Exchange : An Examination of Analyst Price Forecasts ", Contemporary Accounting Research 7, 2 (printemps 1991) : 323-346.

Brown, L. D. et J. C. Y. Han, "The Impact of Annual Earnings Announcements on Convergence of Beliefs ", The Accounting Review 87, 4, (1992) : 862-875.

Brown, P., G. Foster et E. Noreen, Security Analyst Multi-Year Forecasts and the Capital Market, Studies in Accounting Research \#21, American Accounting Association (1985).

Chandra, R. et B.V. Balachandran, "A Synthesis of Alternative Testing Procedures for Event Studies ", Contemporary Accounting Research 6, 2 (1990) : 611-40.

Cragg, J. et B. Malkiel, Expectations and the Structure of Share Prices, Chicago : University of Chicago Press (1982).

Dimson, E. et P. Marsh, "An Analysis of Brokers' and Analysts' Unpublished Forecasts of UK Stocks Returns ", The Journal of Finance (décembre 1984) : 1257-1292. 
Elton, E.J., M.J. Gruber et M.N. Gultekin, «Professional Expectations Accuracy and Diagnosis of Errors ", Journal of Financial and Quantitative Analysis (décembre 1984) : 351-363.

Givoly, D. et J. Lakonishok, "Divergence of Earnings Expectations : The Effect on Stock Market Response to Earnings Signals ", in E. Dimson (ed), Stock Market Anomalies, Cambridge University Press (1988) : 272-289.

Hellwig, M., "On the Aggregation of Information In Competitive Markets ", Journal of Economic Theory 22 (1980) : 477-98.

Holthausen, R. et R. Verrecchia, "The Effect of Informedness and Consensus on Price and Volume Behavior ", The Accounting Review (janvier 1990) : 191-208.

Kazemi, H.B., "Dispersion of Beliefs, Asset Prices, and Noisy Aggregation of Information ", The Financial Review (février 1991): 1-13.

Kim, O. et R.E. Verrecchia, Market Reaction to Anticip ated Announcements, Journal of Financial Economics 30 (1991a) : 273-309.

Kim, O. et R.E. Verrecchia, Trading Volume and Price Reactions to Public Announcements, Journal of Accounting Research 29 (1991b) : 302-321.

L'Her, J.F. et J.M. Suret, " The Reaction of Canadian Securities to Revisions of Earnings Forecasts ", Contemporary Accounting Research (printemps 1991) : 378-406.

Merton, R.C. «Presidential Address : A Simple Model of Capital Market Equilibrium with Incomplete Information " The Journal of Finance 42, 3 (1987) : 483-510.

Morse, D., J. Stephan et E.K. Stice, " Earnings Annoucement and the Convergence (or Divergence) of Beliefs ", The Accounting Review (avril 1991) : 376-388.

O'Brien, P., "Analysts' Forecasts as Earnings Expectations ", Journal of Accounting and Economics (janvier 1988) : 53-83.

Ou, G. et D. Penman, "Accounting Measurement, Price-Earnings Ratio and the Information Content of Security Prices ", Journal of Accounting Research (1990): 111-152.

Peterson, D. et P. Peterson, "Divergence of Opinion and Return ", Journal of Financial Research (été 1982a) : 125-34.

Peterson, D. et P. Peterson, "The Effect of Changing Expectations upon Stock Returns ", Journal of Financial and Quantitative Analysis (décembre 1982b) : 799-811.

Stickel, S.E., "Common Stock Returns Surronding Earnings Forecast Revision : More Puzzling Evidence ", The Accounting Review (avri1 1991) : 402-416. 
Tableau 1 : Évolution de la couverture des titres canadiens par I/B/E/S de janvier 1976 à mai 1994.

Nombre de titres, de prévisions, d'analystes financiers par titre en moyenne, ainsi que de révisions à la hausse et à la baisse pour les mois de décembre de chaque année. Répartition du nombre de titres par catégorie construite par rapport au nombre d'analystes financiers suivant les titres.

\begin{tabular}{|c|c|c|c|c|c|c|c|c|c|c|}
\hline \multirow[t]{2}{*}{ Mois } & \multirow[t]{2}{*}{$\begin{array}{l}\text { Nombre de } \\
\text { titres suivis }\end{array}$} & \multirow[t]{2}{*}{$\begin{array}{c}\text { Nombre de } \\
\text { prévisions }\end{array}$} & \multirow{2}{*}{$\begin{array}{c}\text { Nombre moyen } \\
\text { d'analystes par } \\
\text { titre }\end{array}$} & \multicolumn{2}{|c|}{ Nombre de révisions } & \multicolumn{5}{|c|}{$\begin{array}{l}\text { Répartition du nombre des titres en fonction du } \\
\text { nombre d'analystes qui les suivent }\end{array}$} \\
\hline & & & & Hausse & Baisse & 1 & 2 à 5 & 6 à 11 & 12 à 20 & plus de 20 \\
\hline 7601 & 15 & 56 & 3.7 & na & na & 9 & 2 & 3 & 1 & 0 \\
\hline 7612 & 12 & 56 & 4.7 & na & na & 3 & 5 & 3 & 1 & 0 \\
\hline 7712 & 23 & 70 & 3.0 & na & na & 12 & 8 & 1 & 2 & 0 \\
\hline 7812 & 43 & 130 & 3.0 & na & na & 20 & 20 & 0 & 3 & 0 \\
\hline 7912 & 43 & 155 & 3.6 & na & na & 19 & 17 & 2 & 5 & 0 \\
\hline 8012 & 41 & 171 & 4.2 & na & na & 14 & 20 & 2 & 5 & 0 \\
\hline 8112 & 49 & 252 & 5.1 & na & na & 8 & 29 & 7 & 4 & 1 \\
\hline 8212 & 54 & 248 & 4.6 & na & na & 13 & 29 & 6 & 5 & 1 \\
\hline 8312 & 105 & 354 & 3.4 & na & na & 43 & 49 & 6 & 5 & 2 \\
\hline 8412 & 218 & 843 & 3.9 & na & na & 37 & 142 & 31 & 5 & 3 \\
\hline 8512 & 331 & 2513 & 7.6 & 604 & 219 & 31 & 106 & 132 & 54 & 8 \\
\hline 8612 & 385 & 2870 & 7.5 & 550 & 260 & 52 & 121 & 126 & 76 & 10 \\
\hline 8712 & 489 & 3408 & 7.0 & 452 & 349 & 88 & 177 & 120 & 88 & 16 \\
\hline 8812 & 490 & 3712 & 7.6 & 435 & 231 & 103 & 164 & 108 & 79 & 36 \\
\hline 8912 & 456 & 3401 & 7.5 & 633 & 298 & 92 & 152 & 97 & 86 & 29 \\
\hline 9012 & 432 & 3206 & 7.4 & 774 & 297 & 75 & 147 & 115 & 74 & 21 \\
\hline 9112 & 406 & 2851 & 7.0 & 670 & 283 & 64 & 142 & 121 & 63 & 16 \\
\hline 9212 & 402 & 2566 & 6.4 & 256 & 176 & 67 & 156 & 124 & 43 & 12 \\
\hline 9312 & 422 & 2759 & 6.5 & 460 & 291 & 57 & 161 & 142 & 50 & 12 \\
\hline 9405 & 458 & 2985 & 6.5 & 494 & 493 & 69 & 176 & 146 & 57 & 10 \\
\hline
\end{tabular}

Source: base de données récapitulative $I / B / E / S$, version de juin 1994 . 
Tableau 2: Origine des prévisions de bénéfices en juin 1993 suivant les courtiers et leur pays d'origine.

Les observations sont classées en ordre décroissant du nombre de prévisions réalisées.

\begin{tabular}{|c|c|c|c|c|c|c|c|}
\hline $\begin{array}{c}\text { Numéro } \\
\text { du courtier }\end{array}$ & $\begin{array}{l}\text { Nombre de } \\
\text { prévisions }\end{array}$ & $\begin{array}{c}\text { Nombre } \\
\text { d'analystes }\end{array}$ & Pays & $\begin{array}{c}\text { Numéro } \\
\text { du courtier }\end{array}$ & $\begin{array}{l}\text { Nombre de } \\
\text { prévisions }\end{array}$ & $\begin{array}{c}\text { Nombre } \\
\text { d'analystes }\end{array}$ & Pays \\
\hline 86 & 216 & 19 & $\mathrm{Cdn}$ & 272 & 9 & 5 & U.S. \\
\hline 41 & 205 & 17 & Cdn & 162 & 9 & 4 & U.S. \\
\hline 249 & 171 & 10 & Cdn & 97 & 7 & 5 & U.S. \\
\hline 202 & 171 & 15 & Cdn & 208 & 6 & 3 & U.S. \\
\hline 166 & 153 & 12 & $\mathrm{Cdn}$ & 221 & 6 & 4 & U.S. \\
\hline 191 & 150 & 14 & Cdn & 355 & 5 & 2 & U.S. \\
\hline 333 & 149 & 12 & $\mathrm{Cdn}$ & 133 & 5 & 1 & U.S. \\
\hline 307 & 148 & 13 & Cdn & 233 & 4 & 2 & U.S. \\
\hline 104 & 138 & 11 & Cdn & 91 & 4 & 2 & U.S. \\
\hline 40 & 126 & 8 & $\mathrm{Cdn}$ & 27 & 4 & 3 & U.S. \\
\hline 35 & 117 & 8 & Cdn & 122 & 4 & 3 & U.S. \\
\hline 79 & 97 & 8 & Cdn & 77 & 4 & 1 & U.S. \\
\hline 183 & 83 & 5 & Cdn & 248 & 4 & 2 & U.S. \\
\hline 75 & 78 & 6 & Cdn & 58 & 4 & 2 & U.S. \\
\hline 457 & 76 & 6 & $\mathrm{Cdn}$ & 88 & 4 & 3 & U.S. \\
\hline 185 & 74 & 9 & Cdn & 577 & 3 & 1 & U.S. \\
\hline 247 & 68 & 5 & Cdn & 13 & 3 & 3 & U.S. \\
\hline 589 & 64 & 5 & Cdn & 83 & 3 & 2 & U.S. \\
\hline 605 & 45 & na & Cdn & 31 & 3 & 3 & U.S. \\
\hline 351 & 32 & 3 & U.S. & 628 & 3 & 1 & U.S. \\
\hline 119 & 25 & 10 & Cdn & 238 & 3 & 1 & U.S. \\
\hline 256 & 19 & 8 & U.S. & 300 & 3 & 2 & U.S. \\
\hline 554 & 15 & 1 & U.S. & 299 & 2 & 2 & U.S. \\
\hline 268 & 15 & 5 & $\mathrm{Cdn}$ & 501 & 2 & 2 & U.S. \\
\hline 153 & 14 & 8 & U.S. & 273 & 2 & 2 & U.S. \\
\hline 198 & 14 & 6 & U.S. & 551 & 2 & 2 & U.S. \\
\hline 629 & 13 & 1 & U.S. & 94 & 2 & 2 & U.S. \\
\hline 84 & 13 & 9 & U.S. & 246 & 2 & 2 & U.S. \\
\hline 562 & 12 & 1 & U.S. & 206 & 2 & 2 & U.S. \\
\hline 68 & 11 & 4 & U.S. & 163 & 2 & 2 & U.S. \\
\hline 257 & 11 & 6 & U.S. & 181 & 2 & 1 & U.S. \\
\hline 17 & 10 & 5 & U.S. & 21 & 2 & 2 & U.S. \\
\hline 204 & 10 & 4 & U.S. & 8 & 2 & 2 & U.S. \\
\hline 207 & 10 & 7 & U.S. & 36 & 2 & 2 & U.S. \\
\hline 295 & 10 & 4 & U.S. & \multirow{3}{*}{$\begin{array}{l}40 \text { courtiers } \\
\text { suivant un } \\
\text { seul titre }\end{array}$} & 40 & 40 & U.S. \\
\hline 581 & 10 & 3 & U.S. & & & & \\
\hline 228 & 9 & 3 & U.S. & & & & \\
\hline Total Cdn & 2369 & 198 & & & & & \\
\hline Total U.S. & 213 & 73 & & Total U.S. & 164 & 118 & \\
\hline Total & 2582 & 271 & & Total & 164 & 118 & \\
\hline
\end{tabular}


Tableau 3 : Évolution de la moyenne et de la médiane du consensus (PM), de la dispersion (ET) et des variations mensuelles de ces variables $\left(\Delta^{*} A M\right.$ et $\left.\Delta^{*} D I S P\right)$ au cours de l'exercice financier. Les 21642 observations sont regroupées par mois d'exercice financier.

\begin{tabular}{|c|c|c|c|c|c|c|c|c|c|c|c|}
\hline \multirow[t]{2}{*}{$\begin{array}{l}\text { Mois de } \\
\text { l'exercice }\end{array}$} & \multirow[t]{2}{*}{$\begin{array}{c}\# \\
\text { Obs. }\end{array}$} & \multicolumn{2}{|c|}{$\begin{array}{l}\text { Prévision de BPA } \\
\text { (PM) }\end{array}$} & \multicolumn{3}{|c|}{$\begin{array}{c}\text { Révision normalisée } \\
\qquad\left(\Delta^{*} A M\right)\end{array}$} & \multicolumn{2}{|c|}{$\begin{array}{l}\text { Écart-type des } \\
\text { prévisions (ET) }\end{array}$} & \multicolumn{3}{|c|}{$\begin{array}{c}\text { Changements de la dispersion } \\
\text { ( } \Delta^{*} \text { DISP) }\end{array}$} \\
\hline & & Moyenne & Médiane & $\begin{array}{c}\text { Moyenne } \\
\%\end{array}$ & $\begin{array}{l}\text { Médiane } \\
\%\end{array}$ & $\begin{array}{l}\% \mathrm{de} \\
\text { révisions nulles }\end{array}$ & Moyenne & Médiane & $\begin{array}{l}\text { Moyenne } \\
\%\end{array}$ & $\begin{array}{c}\text { Médiane } \\
\%\end{array}$ & $\begin{array}{c}\% \text { de } \\
\text { variations nulles }\end{array}$ \\
\hline 1 & 1787 & 0.851 & 0.690 & & & & 0.153 & 0.100 & & & \\
\hline 2 & 1842 & 0.943 & 0.780 & 0.470 & 0.000 & 20.52 & 0.186 & 0.110 & 0.190 & 0.000 & 27.42 \\
\hline 3 & 1840 & 1.041 & 0.860 & 0.650 & 0.100 & 12.07 & 0.216 & 0.140 & 0.230 & 0.050 & 18.15 \\
\hline 4 & 1840 & 1.060 & 0.900 & -0.030 & 0.000 & 14.95 & 0.220 & 0.140 & 0.020 & 0.000 & 21.36 \\
\hline 5 & 1871 & 1.053 & 0.890 & -0.140 & -0.050 & 13.79 & 0.211 & 0.140 & -0.050 & 0.000 & 21.38 \\
\hline 6 & 1755 & 1.048 & 0.870 & -0.230 & -0.060 & 15.61 & 0.205 & 0.130 & -0.060 & 0.000 & 24.56 \\
\hline 7 & 1773 & 1.022 & 0.850 & -0.220 & -0.060 & 19.74 & 0.196 & 0.130 & -0.070 & 0.000 & 28.37 \\
\hline 8 & 1786 & 0.969 & 0.810 & -0.230 & -0.070 & 18.92 & 0.188 & 0.130 & -0.030 & 0.000 & 26.82 \\
\hline 9 & 1783 & 0.943 & 0.770 & -0.300 & -0.070 & 17.61 & 0.180 & 0.120 & -0.040 & 0.000 & 25.24 \\
\hline 10 & 1793 & 0.920 & 0.750 & -0.240 & -0.040 & 22.81 & 0.173 & 0.120 & -0.060 & 0.000 & 30.28 \\
\hline 11 & 1784 & 0.897 & 0.740 & -0.320 & -0.070 & 16.65 & 0.168 & 0.110 & -0.030 & 0.000 & 25.34 \\
\hline 12 & 1788 & 0.870 & 0.720 & -0.330 & -0.060 & 19.41 & 0.156 & 0.100 & -0.070 & 0.000 & 26.06 \\
\hline Total & 21642 & 0.983 & 0.810 & 0.083 & -0.034 & 17.43 & 0.194 & 0.000 & -0.008 & 0.000 & 25.87 \\
\hline
\end{tabular}

* indique que la moyenne est statistiquement différente de 0 au seuil de signification de $5 \%$; la matrice des coefficients de corrélation entre les variables explicatives est la suivante (niveau de signification entre parenthèses) :

\begin{tabular}{cccc}
\hline & $\Delta^{*} D I S P$ & NBA & CV \\
$\Delta^{*} A M$ & $0.059(0.0001)$ & $0.013(0.0985)$ & $-0.0882(0.0001)$ \\
$\Delta^{*} D I S P$ & & $-0.021(0.0079)$ & $0.0732(0.0001)$ \\
NBA & & & $-0.0223(0.0046)$ \\
\hline
\end{tabular}


Figure 1 : Fractiles de la distribution des changements dans la dispersion des prévisions des analystes financiers $\left(\Delta^{*} D I S P\right)$ en fonction des valeurs médianes des déciles de la distributions des révisions $\left(\Delta^{*} A M\right)$

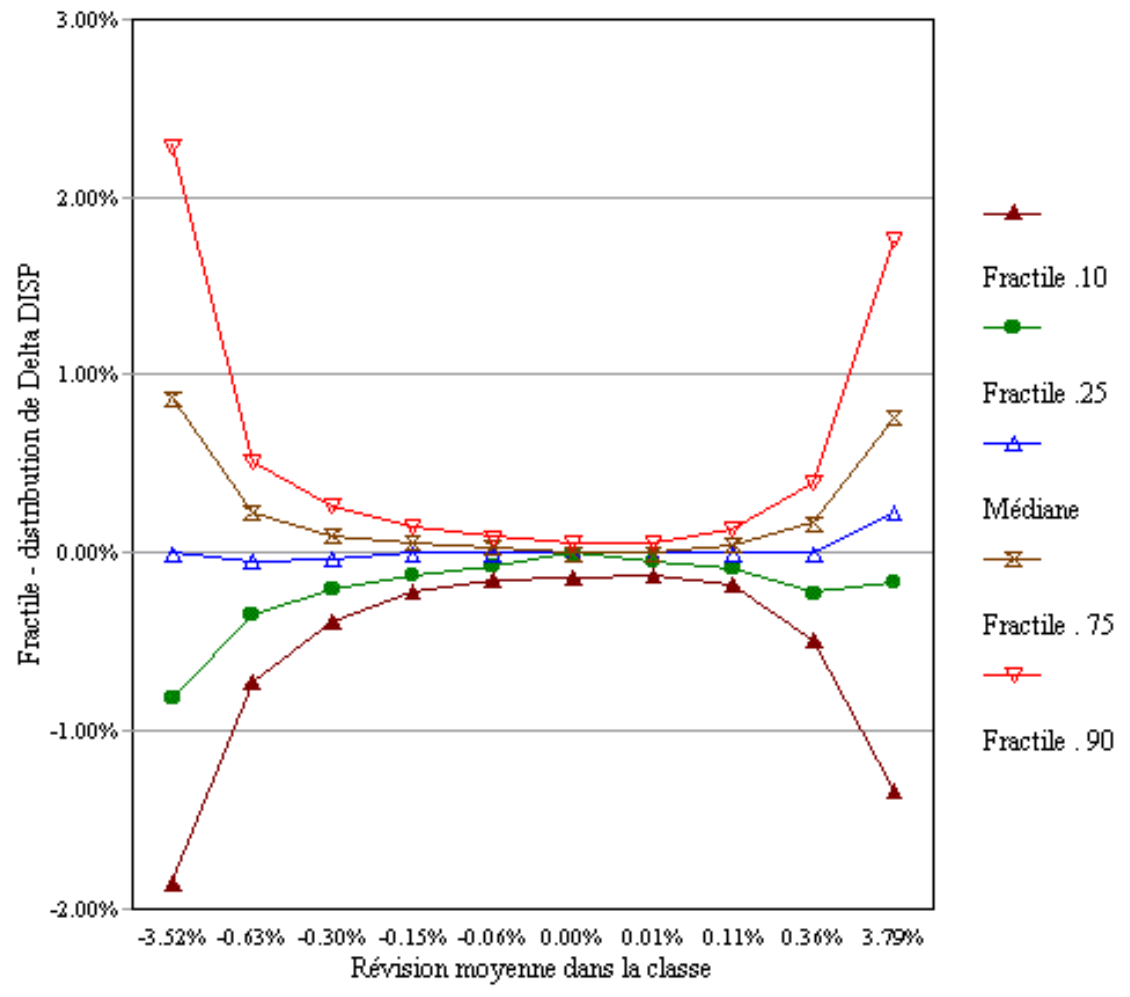


Tableau 4 : Coefficients moyens $\alpha_{\mathrm{i}}(\mathrm{i}=1,2)$ et tests statistiques ${ }^{\mathrm{a}}$ correspondant au modèle : $R_{j t}-\alpha_{0 t}+\alpha_{1 t} \Delta^{*} A M_{j t}+\alpha_{2 t} \Delta^{*} D I S P_{j t}+\varepsilon_{j t} \quad ;$ t allant de janvier 1985 à mai 1994. Le rendement est mesuré par le rendement résiduel calculé à partir du CAPM et du modèle de rendements ajustés pour le marché. Res_1, Res0 et Res1 représentent respectivement les résidus calculés un mois avant, le mois même et un mois après les changements dans la distribution des $P A F$. Les observations du mois de janvier sont exclues.

\begin{tabular}{|c|c|c|c|c|c|}
\hline Modèle & Var. dépendante & $\Delta^{*} \mathrm{AM}$ & $\Delta^{*} \mathrm{DISP}$ & $\mathrm{CV}$ & pseudo $\mathrm{R}^{2}$ \\
\hline \multicolumn{6}{|c|}{ Volet A: Estimation des coefficients moyens, ensemble de l'échantillon } \\
\hline CAPM & Res_1 & $0.970(7.614)^{*}$ & $-0.370(-2.126)^{*}$ & & 0.051 \\
\hline CAPM & Res0 & $0.678(6.901) *$ & $-0.314(-2.126)^{*}$ & & 0.036 \\
\hline CAPM & Res1 & $0.414(4.286)^{*}$ & $-0.481(-3.609)^{*}$ & & 0.028 \\
\hline \multicolumn{6}{|c|}{ Volet B: Estimation Huang Litzenberger, ensemble de l'échantillon } \\
\hline CAPM & Res_1 & $0.785(15.02)^{*}$ & $-0.320(-3.40)^{*}$ & & 0.051 \\
\hline CAPM & Res0 & $0.480(9.20)^{*}$ & $-0.241(-2.55)^{*}$ & & 0.036 \\
\hline CAPM & Res1 & $0.301(5.79)^{*}$ & $-0.372(-3.96)^{*}$ & & 0.028 \\
\hline \multicolumn{6}{|c|}{ Volet C: Estimation des coefficients moyens, ensemble de l'échantillon } \\
\hline M Marché & Res_1 & $0.898(7.732)^{*}$ & $-0.265(-1.658)$ & & \\
\hline M Marché & Res0 & $0.559(6.219)^{*}$ & $-0.272(-1.843)$ & & \\
\hline M Marché & Res1 & $0.388(4.134)^{*}$ & $-0.462(-3.484)^{*}$ & & \\
\hline \multicolumn{6}{|c|}{ Volet D: Estimation des coefficients moyens, titres suivis par moins de 12 analystes } \\
\hline CAPM & Res_1 & $0.904(6.122) *$ & $-0.313(-1.535)$ & $-0.001(-0.477)$ & \\
\hline CAPM & Res0 & $0.778(5.296)^{*}$ & $-0.398(-2.093)^{*}$ & $-0.001(-0.800)$ & \\
\hline CAPM & Res1 & $0.356(2.513) *$ & $-0.4058(-2.264) *$ & $0.001(0.476)$ & \\
\hline \multicolumn{6}{|c|}{ Estimation des coefficients moyens, titres suivis par 12 analystes et plus } \\
\hline CAPM & Res_1 & $1.410(5.696) *$ & $-1.157(-2.487)^{*}$ & $-0.002(-0.425)$ & \\
\hline CAPM & Res0 & $0.934(3.684) *$ & $-0.310(0.720)$ & $0.003(-0.920)$ & \\
\hline CAPM & Res1 & $0.446(2.524) *$ & $-0.514(-1.567)$ & $-0.001(-0.420)$ & \\
\hline
\end{tabular}

* indique que le test est significatif au seuil de $5 \%$;

Suivant HL, la valeur $t$ se calcule à partir du coefficient moyen et de sa variance estimés par :

$$
\bar{\alpha}_{\mathrm{i}}=\sum_{\mathrm{t}=1}^{\mathrm{T}} \mathrm{w}_{\mathrm{t}} \hat{\alpha}_{\mathrm{it}} \text { où } \quad \mathrm{w}_{\mathrm{t}}=\frac{\operatorname{Var}^{-1}\left(\hat{\alpha}_{\mathrm{it}}\right)}{\sum_{\mathrm{t}=1}^{\mathrm{T}} \operatorname{Var}^{-1}\left(\hat{\alpha}_{\mathrm{it}}\right)} \text { et } \operatorname{Var}\left(\hat{\alpha}_{\mathrm{i}}\right)=\sum_{\mathrm{t}=1}^{\mathrm{T}} \mathrm{w}_{\mathrm{t}}^{2} \operatorname{Var}\left(\hat{\alpha}_{\mathrm{it}}\right)
$$

Le pseudo- $\mathrm{R}^{2}$ est calculé de la façon suivante (voir, par exemple, Janagathan et Wang, 1994) :

$$
\mathrm{R}^{2}=1-\sum_{\mathrm{t}=1}^{\mathrm{T}} \mathrm{SCE}_{\mathrm{t}} / \sum_{\mathrm{t}=1}^{\mathrm{T}} \mathrm{SCT}_{\mathrm{t}}
$$

où $\mathrm{SCE}$ et $\mathrm{SCT}$ représentent respectivement les sommes des carrés expliqués et des carrés totaux. Dans la méthode des coefficients moyens, $\mathrm{w}_{\mathrm{t}}=1 / \mathrm{T}$, ou $\mathrm{T}$ est le nombre de régressions mensuelles. 
Figure 2: Rendements excédentaires cumulés de portefeuilles de titres composés en fonction des changements dans le consensus $\left(\Delta^{*} A M\right)$ et la dispersion $\left(\Delta^{*} D I S P\right)$ des prévisions des analystes financiers.

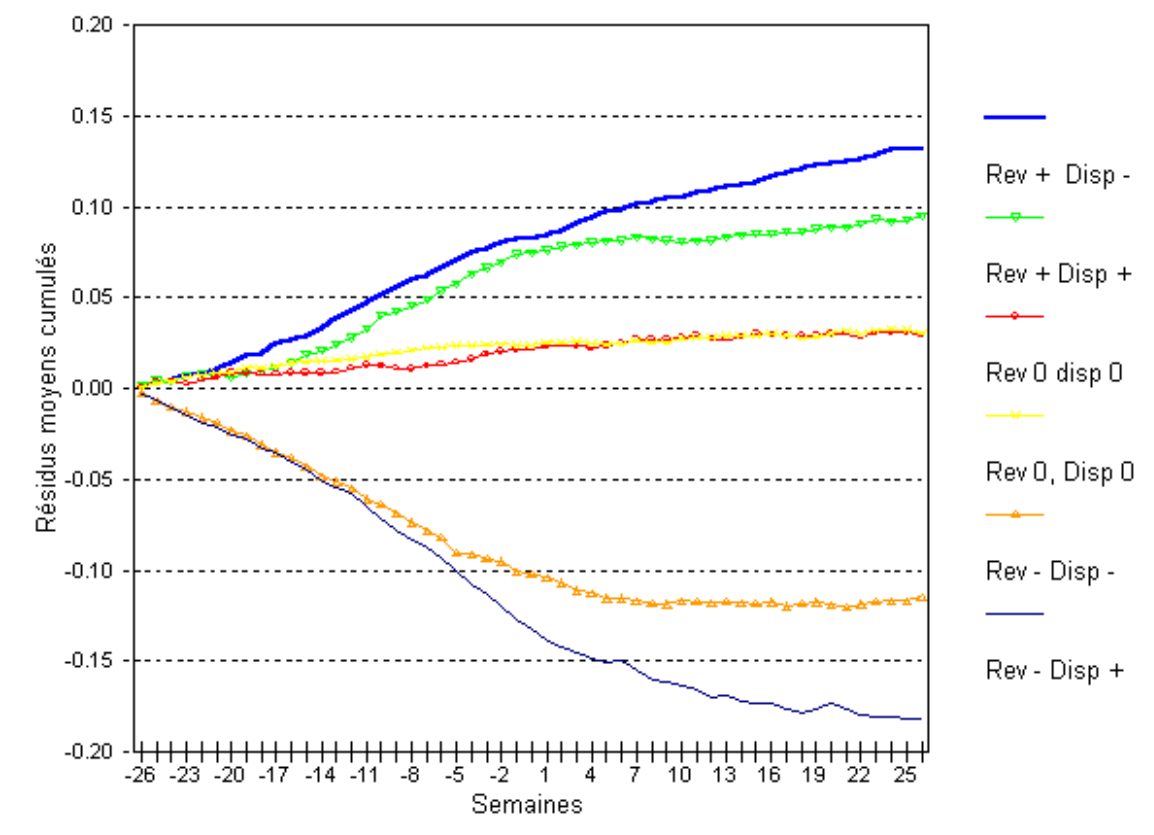

1) Dans le CAPM, le rendement théorique d'un titre i est égal à $E\left(R_{i t}\right)=R_{f t}+\beta_{i}\left[E\left(R_{m t}\right)-R_{f t}\right]$, où $R_{f t}$ désigne le taux de rendement mensuel sur l'actif sans risque. $E\left(R_{m t}\right)-R_{f t}$ représente la prime de risque et $\beta_{\mathrm{i}}$ le risque systématique du titre i. Le résidu est donné par : $e_{i t}=\left(R_{i t}-R_{f t}\right)-\beta_{i}\left[R_{m t}-R_{f f}\right]$, où $\hat{\beta}_{i}$ désigne l'estimateur du risque systématique du titre i. L'estimateur de l'ordonnée à l'origine $\hat{\alpha}_{\text {it }}$, n'apparaît pas dans le calcul du résidu (Ross, 1976, Brown, Foster et Noreen (1985: 79).

2) Dans le modèle de marché, le rendement théorique d'un titre i est égal à: $E\left(R_{i t}\right)=\alpha_{i}+\beta_{i} E\left(R_{m t}\right)$. $\alpha_{i}$ et $\beta_{\mathrm{i}}$ sont les coefficients de la régression des rendements du titre par rapport à ceux du marché, calculés à l'aide des 80 rendements hebdomadaires qui précèdent la semaine -26, (la semaine 0 étant celle de l'événement). Le résidu $\mathrm{e}_{\mathrm{it}}$ est donné par : $\mathrm{R}_{\mathrm{it}}-\left(\hat{\alpha}_{\mathrm{i}}+\hat{\beta}_{\mathrm{i}} \mathrm{R}_{\mathrm{mt}}\right)$. 3) Dans le modèle de rendements ajustés pour le marché, $E\left(R_{i t}\right)=E\left(R_{j t}\right)=E\left(R_{m t}\right)$, soit le rendement du portefeuille de marché. Le résidu est la différence: $\mathrm{R}_{\mathrm{it}}-\mathrm{R}_{\mathrm{mt}}$. 
Tableau 5 : Résidus moyens et tests statistiques autour des dates de changements dans le consensus $\left(\Delta^{*} A M\right)$ et la dispersion $\left(\Delta^{*} D I S P\right)$ des prévisions des analystes financiers. Résidus moyens cumulés à partir de la date de révisions des prévisions des analystes financiers et tests statistiques correspondants. L'analyse porte sur l'ensemble des observations et la récurrence n'est pas traitée.

\begin{tabular}{|c|c|c|c|c|c|c|c|c|c|c|c|c|c|}
\hline \multicolumn{7}{|c|}{ Résidus moyen autour de la date de révision } & \multicolumn{7}{|c|}{ Résidus moyens cumulés à partir de la date de révision } \\
\hline \multirow[b]{2}{*}{ Sem } & \multicolumn{3}{|c|}{ Révisions en hausse } & \multicolumn{3}{|c|}{ Révisions en baisse } & \multirow[b]{2}{*}{ Sem } & \multicolumn{3}{|c|}{ Révisions en hausse } & \multicolumn{3}{|c|}{ Révisions en baisse } \\
\hline & $\begin{array}{c}\text { RM } \\
\text { Disp - }\end{array}$ & val t & $\begin{array}{c}\text { RM } \\
\text { Disp+ } \\
\end{array}$ & $\begin{array}{c}\text { RM } \\
\text { Disp - } \\
\end{array}$ & val $\mathrm{t}$ & $\begin{array}{c}\text { RM } \\
\text { Disp+ } \\
\end{array}$ & & $\begin{array}{l}\text { RMC } \\
\text { Disp - }\end{array}$ & val $\mathrm{t}$ & $\begin{array}{c}\text { RMC } \\
\text { Disp + }\end{array}$ & $\begin{array}{l}\text { RMC } \\
\text { Disp - } \\
\end{array}$ & val t & $\begin{array}{l}\text { RM.C } \\
\text { Disp + }\end{array}$ \\
\hline-13 & 0.006 & 1.23 & 0.003 & -0.003 & 0.49 & -0.004 & 0 & 0.001 & 0.01 & 0.001 & -0.002 & 1.79 & -0.006 \\
\hline-12 & 0.004 & -0.19 & 0.004 & -0.004 & -0.49 & -0.002 & 1 & 0.003 & 0.22 & 0.002 & -0.003 & $2.59 *$ & -0.012 \\
\hline-11 & 0.005 & -0.06 & 0.005 & -0.006 & 0.84 & -0.008 & 2 & 0.005 & 0.19 & 0.004 & -0.006 & $2.39 *$ & -0.016 \\
\hline-10 & 0.004 & -1.28 & 0.007 & -0.003 & 1.78 & -0.007 & 3 & 0.010 & 0.93 & 0.005 & -0.011 & 1.70 & -0.018 \\
\hline-9 & 0.005 & 0.97 & 0.002 & -0.005 & 0.46 & -0.006 & 4 & 0.013 & 1.19 & 0.006 & -0.012 & $1.97 *$ & -0.022 \\
\hline-8 & 0.005 & 0.74 & 0.003 & -0.005 & 0.11 & -0.005 & 5 & 0.015 & 1.42 & 0.007 & -0.015 & 1.58 & -0.024 \\
\hline-7 & 0.002 & -0.64 & 0.003 & -0.005 & 0.04 & -0.005 & 6 & 0.017 & 1.45 & 0.008 & -0.015 & 1.37 & -0.023 \\
\hline-6 & 0.005 & -0.24 & 0.005 & -0.004 & 0.67 & -0.005 & 7 & 0.019 & 1.52 & 0.009 & -0.016 & 1.78 & -0.028 \\
\hline-5 & 0.004 & -0.06 & 0.004 & -0.008 & -0.50 & -0.007 & 8 & 0.021 & 1.86 & 0.008 & -0.018 & 2.27 * & -0.033 \\
\hline-4 & 0.004 & -0.37 & 0.005 & -0.001 & $2.77 *$ & -0.007 & 9 & 0.023 & $2.06^{*}$ & 0.007 & -0.018 & $2.28 *$ & -0.035 \\
\hline-3 & 0.003 & -0.36 & 0.004 & -0.002 & 1.47 & -0.006 & 10 & 0.023 & $2.12 *$ & 0.007 & -0.016 & $2.71 *$ & -0.037 \\
\hline-2 & 0.003 & -0.02 & 0.003 & -0.002 & $2.05^{*}$ & -0.006 & 11 & 0.026 & $2.34 *$ & 0.007 & -0.016 & $2.85^{*}$ & -0.039 \\
\hline-1 & 0.002 & -1.37 & 0.005 & -0.006 & 0.63 & -0.007 & 12 & 0.027 & $2.33^{*}$ & 0.007 & -0.017 & 3.13* & -0.043 \\
\hline 0 & 0.001 & 0.01 & 0.001 & -0.002 & 1.78 & -0.006 & 13 & 0.029 & $2.27^{*}$ & 0.009 & -0.016 & $2.99 *$ & -0.042 \\
\hline 1 & 0.002 & 0.30 & 0.002 & -0.002 & 1.88 & -0.006 & 14 & 0.031 & $2.26^{*}$ & 0.011 & -0.017 & $3.06 *$ & -0.045 \\
\hline 2 & 0.002 & 0.02 & 0.002 & -0.003 & 0.47 & -0.004 & 15 & 0.032 & $2.23 *$ & 0.011 & -0.018 & $3.17 *$ & -0.047 \\
\hline 3 & 0.004 & 1.53 & 0.001 & -0.004 & -0.73 & -0.003 & 16 & 0.035 & $2.46^{*}$ & 0.011 & -0.017 & $3.12 *$ & -0.047 \\
\hline 4 & 0.003 & 0.80 & 0.001 & -0.001 & 0.99 & -0.004 & 17 & 0.037 & $2.47 *$ & 0.012 & -0.019 & $3.14 *$ & -0.050 \\
\hline 5 & 0.003 & 0.81 & 0.001 & -0.003 & -0.52 & -0.002 & 18 & 0.039 & $2.62^{*}$ & 0.012 & -0.018 & $3.40 *$ & -0.052 \\
\hline 6 & 0.001 & 0.36 & 0.000 & 0.000 & -0.25 & 0.001 & 19 & 0.041 & $2.63^{*}$ & 0.014 & -0.017 & $3.16^{*}$ & -0.050 \\
\hline 7 & 0.003 & 0.47 & 0.002 & -0.002 & 1.40 & -0.005 & 20 & 0.043 & $2.60 \%$ & 0.015 & -0.018 & $2.71 *$ & -0.047 \\
\hline 8 & 0.002 & 1.29 & -0.001 & -0.001 & 1.80 & -0.005 & 21 & 0.044 & $2.65^{*}$ & 0.015 & -0.020 & $2.79 *$ & -0.050 \\
\hline 9 & 0.001 & 0.92 & -0.001 & -0.000 & 0.37 & -0.001 & 22 & 0.044 & $2.49^{*}$ & 0.017 & -0.018 & $3.17^{*}$ & -0.053 \\
\hline 10 & 0.001 & 0.53 & -0.000 & 0.002 & 1.79 & -0.002 & 23 & 0.047 & $2.42 *$ & 0.019 & -0.016 & $3.31 *$ & -0.054 \\
\hline 11 & 0.003 & 1.08 & 0.000 & -0.000 & 0.90 & -0.002 & 24 & 0.049 & $2.70^{*}$ & 0.018 & -0.016 & $3.26^{*}$ & -0.054 \\
\hline 12 & 0.001 & 0.29 & 0.000 & -0.001 & 1.40 & -0.004 & 25 & 0.050 & $2.63^{*}$ & 0.019 & -0.016 & $3.34 *$ & -0.055 \\
\hline 13 & 0.002 & 0.09 & 0.002 & 0.001 & -0.11 & 0.001 & 26 & 0.050 & $2.35^{*}$ & 0.021 & -0.014 & $3.41 *$ & -0.055 \\
\hline
\end{tabular}


Figure 3 : Rendements excédentaires cumulés de portefeuilles de titres composés en fonction de la révision et du changement dans la dispersion des prévisions des analystes financiers, pour les titres suivis par moins de 12 analystes.

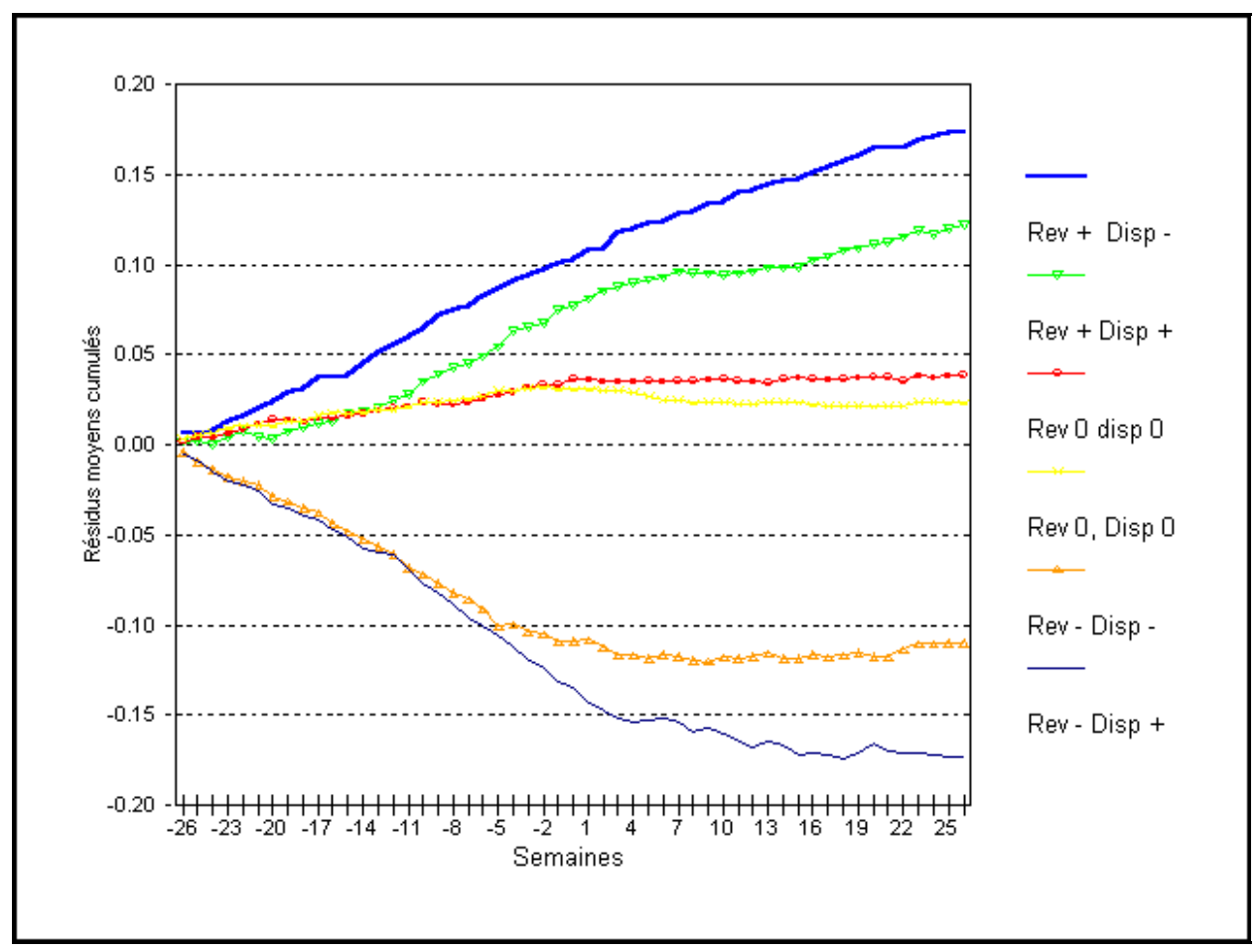


Figure 4: Rendements excédentaires cumulés de portefeuilles de titres composés en fonction de la révision et du changement dans la dispersion des prévisions des analystes financiers, pour les titres suivis par 12 analystes et plus.

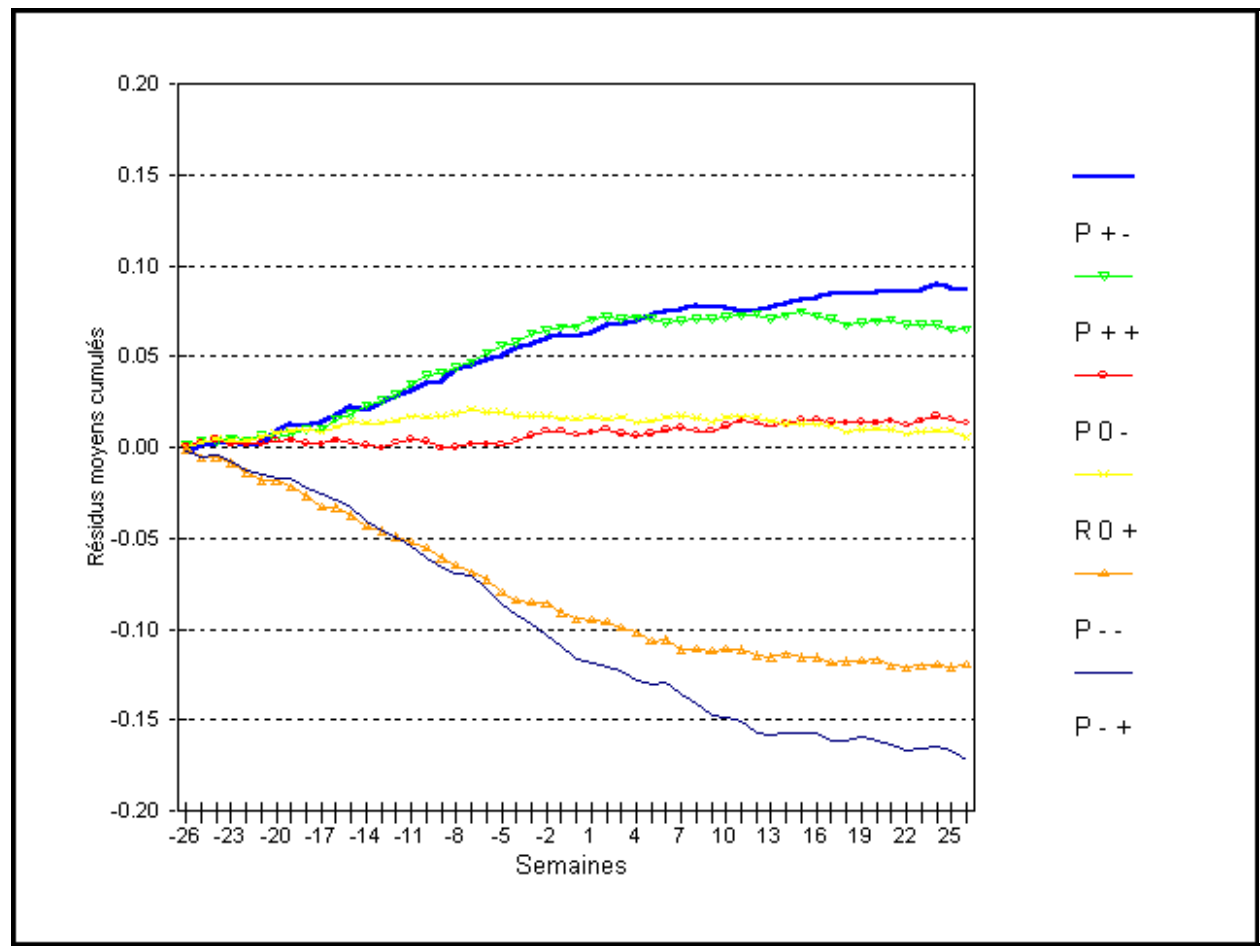


Figure 5 : Rendements excédentaires cumulés de portefeuilles de titres composés en fonction de la révision et du changement dans la dispersion des prévisions des analystes financiers, pour l'ensemble des titres, lorsque les situations de récurrence sont traitées. Les révisions précédées par une autre révision qui se classe dans le même fractile sont omises.

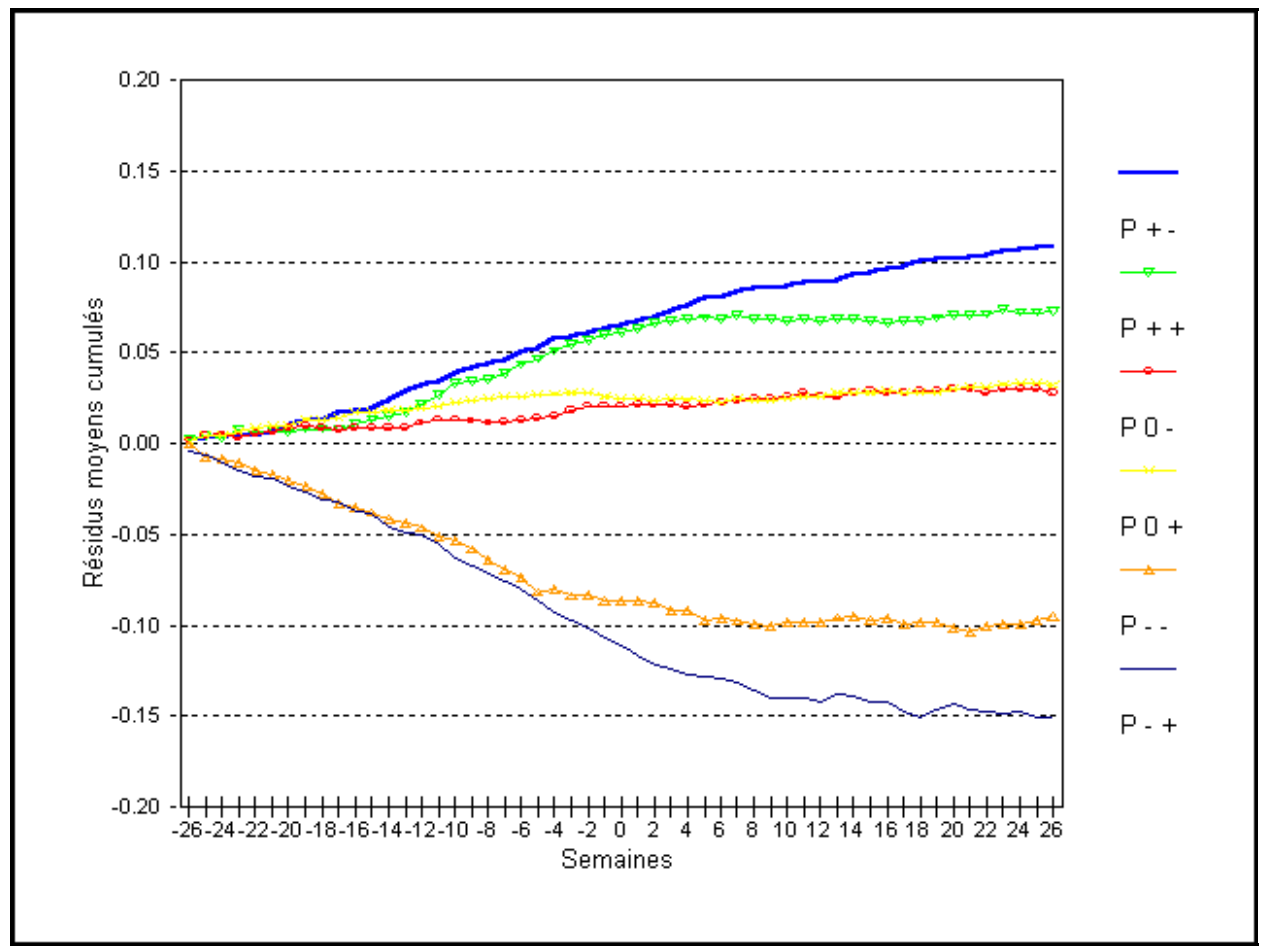


Tableau 6 : Analyse de la sensibilité des résultats de l'analyse résiduelle à l'effet janvier, au nombre d'analystes, au traitement de la récurrence et au choix des modèles et des indices : résidus moyens cumulés et tests statistiques aux semaines $0,6,12$ et 24 , suivant la révision et le changement dans la dispersion.

\begin{tabular}{|c|c|c|c|c|c|c|c|c|c|c|c|c|c|}
\hline \multirow[b]{2}{*}{ Sem } & \multicolumn{3}{|c|}{ Révisions en hausse } & \multicolumn{3}{|c|}{ Révisions en baisse } & \multicolumn{4}{|c|}{ Révisions en hausse } & \multicolumn{3}{|c|}{ Révisions en baisse } \\
\hline & $\begin{array}{l}\text { RMC } \\
\text { Disp - }\end{array}$ & val. $\mathrm{t}$ & $\begin{array}{r}\text { RMC } \\
\text { Disp + }\end{array}$ & $\begin{array}{l}\text { RMC } \\
\text { Disp - }\end{array}$ & val. $\mathrm{t}$ & $\begin{array}{r}\text { RMC } \\
\text { Disp }+\end{array}$ & Sem & $\begin{array}{l}\text { RMC } \\
\text { Disp - }\end{array}$ & val. $\mathrm{t}$ & $\begin{array}{c}\text { RMC } \\
\text { Disp + }\end{array}$ & $\begin{array}{c}\text { RMC } \\
\text { Disp - }\end{array}$ & val. $\mathrm{t}$ & $\begin{array}{c}\text { RMC } \\
\text { Disp + }\end{array}$ \\
\hline \multicolumn{14}{|c|}{ CAPM, Indice TSE TR, ensemble des observations (Rappel) } \\
\hline 0 & 0.001 & 0.01 & 0.001 & -0.002 & 1.79 & -0.006 & 12 & 0.027 & $2.33 *$ & 0.007 & -0.017 & $3.13 *$ & -0.043 \\
\hline 6 & 0.017 & 1.45 & 0.008 & -0.015 & 1.37 & -0.023 & 24 & 0.049 & $2.70^{*}$ & 0.018 & -0.016 & $3.26^{*}$ & -0.054 \\
\hline \multicolumn{14}{|c|}{ Volet A: analyse de l'effet de la composition de l'échantillon } \\
\hline \multicolumn{14}{|c|}{ CAPM, indice TSE TR, mois de janvier omis } \\
\hline 0 & 0.001 & 0.14 & 0.001 & -0.001 & $1.98 *$ & -0.006 & 12 & 0.027 & $2.43 *$ & 0.006 & -0.017 & $3.37 *$ & -0.046 \\
\hline 6 & 0.016 & 1.52 & 0.007 & -0.014 & 1.54 & -0.023 & 24 & 0.049 & $2.69 *$ & 0.017 & -0.016 & $3.32 *$ & -0.054 \\
\hline \multicolumn{14}{|c|}{ CAPM, indice TSE TR, moins de 12 analystes } \\
\hline 0 & 0.003 & 0.01 & 0.003 & 0.000 & 1.20 & -0.003 & 12 & 0.041 & 1.69 & 0.021 & -0.008 & $2.54 *$ & -0.036 \\
\hline 6 & 0.024 & 0.66 & 0.018 & -0.007 & 1.61 & -0.020 & 24 & 0.071 & 1.73 & 0.042 & -0.001 & $2.53 *$ & -0.040 \\
\hline \multicolumn{14}{|c|}{ CAPM, indice TSE TR, 12 analystes et plus } \\
\hline 0 & -0.001 & -0.43 & 0.000 & -0.004 & 1.21 & -0.008 & 12 & 0.013 & 0.49 & 0.007 & -0.024 & $2.29 *$ & -0.048 \\
\hline 6 & 0.012 & 1.17 & 0.003 & -0.015 & .71 & -0.021 & 24 & 0.027 & 1.69 & -0.001 & -0.028 & $2.24 *$ & -0.063 \\
\hline \multicolumn{14}{|c|}{ CAPM, indice TSE TR, après omission des situations de récurrence } \\
\hline 0 & 0.003 & 0.60 & 0.001 & -0.000 & 1.38 & -0.005 & 12 & 0.026 & $2.21 *$ & 0.007 & -0.012 & 1.76 & -0.035 \\
\hline 6 & 0.018 & 1.44 & 0.009 & -0.010 & 1.33 & -0.023 & 24 & 0.045 & $2.78^{*}$ & 0.012 & -0.007 & $1.99^{*}$ & -0.042 \\
\hline \multicolumn{14}{|c|}{ Volet B: analyse de l'effet du choix du modèle générateur des rendements } \\
\hline \multicolumn{14}{|c|}{ Modèle de marché, indice TSE TR } \\
\hline 0 & 0.001 & 0.24 & 0.000 & -0.001 & 1.78 & -0.005 & 12 & 0.026 & $3.21 *$ & -0.001 & -0.009 & $2.99 *$ & -0.034 \\
\hline 6 & 0.016 & $2.09 *$ & 0.003 & -0.010 & 1.27 & -0.018 & 24 & 0.048 & $3.82 *$ & 0.004 & 0.001 & $3.11 *$ & -0.035 \\
\hline \multicolumn{14}{|c|}{ Modèle de rendement moyen du marché, indice TSE TR } \\
\hline 0 & 0.001 & 0.06 & 0.001 & -0.001 & 1.52 & -0.005 & 12 & 0.027 & 1.81 & 0.011 & -0.010 & $3.27 *$ & -0.037 \\
\hline 6 & 0.017 & 1.33 & 0.008 & -0.011 & 1.43 & -0.020 & 24 & 0.051 & $2.38 *$ & 0.023 & -0.007 & $3.16^{*}$ & -0.044 \\
\hline \multicolumn{14}{|c|}{ Volet C: analyse de l'effet du choix de l'indice de marché } \\
\hline \multicolumn{14}{|c|}{ CAPM avec un indice équipondéré composé à partir des seuls titres présents dans l'échantillon } \\
\hline 0 & -0.001 & -0.12 & -0.000 & -0.003 & 1.23 & -0.006 & 12 & 0.001 & 1.76 & -0.015 & -0.042 & $2.63 *$ & -0.065 \\
\hline 6 & 0.004 & 1.22 & -0.004 & -0.027 & 1.04 & -0.034 & 24 & -0.001 & $2.22 *$ & -0.029 & -0.067 & $2.74 *$ & -0.101 \\
\hline \multicolumn{14}{|c|}{ CAPM avec indice global équipondéré, composé à partir de l'ensemble des titres du TSE } \\
\hline 0 & 0.001 & -0.08 & 0.001 & -0.002 & 1.60 & -0.005 & 12 & 0.022 & $2.28 *$ & 0.003 & -0.021 & $3.09 *$ & -0.047 \\
\hline 6 & 0.014 & 1.37 & 0.005 & -0.017 & 1.38 & -0.025 & 24 & 0.040 & $2.57^{*}$ & 0.010 & -0.024 & $3.32 *$ & -0.062 \\
\hline
\end{tabular}




\section{ANNEXE}

\subsection{Une expression du prix d'équilibre}

Les investisseurs qui possèdent un patrimoine initial $W_{j 0}$ entrent sur le marché au temps 0 et à la fin de la période reçoivent la valeur future de leur investissement initial. La richesse initiale de l'investisseur $\mathrm{j}$ est donnée par :

$W_{j 0}=Z_{j} P+D_{j}$, où $Z_{j}$ représente le nombre d'actions achetées par l'investisseur $\mathrm{j}$, alors que $D_{j}$ dénote l'investissement dans l'actif sans risque. La richesse de l'investisseur j en fin de période est donc égale à $W_{j 1}=Z_{j} \tilde{C}+D_{j} R=Z_{j} \tilde{C}+R\left(W_{j 0}-Z_{j} P\right)$.

Si l'on suppose que les fonctions d'utilité des investisseurs sont de la forme exponentielle négative, -exp(- $r_{j}^{-}$ $\left.{ }^{1} W_{1 j}\right)$, où $r_{j}$ désigne la tolérance au risque de l'investisseur $j \mathrm{j}$, le problème d'allocation du patrimoine initial de l'investisseur j entre l'actif risqué et l'actif sans risque est le suivant :

$$
\operatorname{Max} E\left[-\exp \left(-r_{j}^{-1} \tilde{W}_{j 1} \mid Y_{j}, P\right)\right] \equiv \operatorname{Max} E\left(\tilde{W}_{j 1} \mid Y_{j}, P\right)-\frac{r_{j}}{2} \operatorname{Var}\left(\tilde{W}_{j 1} \mid Y_{j}, P\right)
$$

Sous les hypothèses du modèle hybride (distribution normale de la richesse future et fonction d'utilité exponentielle négative), la demande de l'investisseur j pour l'actif risqué s'écrit :

$$
Z_{j}=r_{j} \cdot \frac{E\left(\tilde{C} \mid Y_{j}, P\right)-R P}{\operatorname{Var}\left(\tilde{C} \mid Y_{j}, P\right)}=r_{j} \cdot \frac{f_{j}-R P}{V}
$$

Hellwig (1980) a montré que si le prix d'ÉARB est une fonction mesurable linéaire par rapport aux signaux individuels reçus par les agents informés $Y_{j}$ et par rapport à l'offre nette d'actif risqué, il converge en probabilité vers $P=K_{0}+K_{1} \tilde{C}-K_{2} \tilde{S}$. Le prix de l'actif risqué est alors indépendant des termes d'erreur individuels. II est distribué normalement car par hypothèses, $\tilde{C}$ et $\tilde{S}$ sont distribués normalement. Les propriétés des distributions normales permettent alors d'écrire que l'espérance et la variance conditionnelles du prix de l'actif risqué connaissant l'information privée, $Y_{j}$, et le prix actuel de l'actif risqué, $P$ :

$$
\begin{aligned}
& E\left(\tilde{C} \mid Y_{j}, P\right)=f_{j}=B_{0}+B_{1} Y_{j}+B_{2} P \\
& \operatorname{Var}\left(\tilde{C} \mid Y_{j}, P\right)=V
\end{aligned}
$$

où $\mathrm{B}_{0}, \mathrm{~B}_{1}, \mathrm{~B}_{2}$ et $\mathrm{V}$ sont indépendants de $Y_{\mathrm{j}}$ et de $P$.

Substituant les expressions (A3) dans (A2), la demande de l'investisseur j peut être récrite comme suit :

$$
Z_{j}=r_{j} \frac{B_{0}+B_{1} Y_{j}+B_{2} P-R P}{V}
$$


Cette expression représente la demande optimale de l'investisseur pour l'actif risqué, conditionnelle au contenu informationnel de son signal privé et au prix d'équilibre de l'actif risqué. C'est une fonction linéaire de $Y_{j}$ et de $P$.

Comme il existe un continuum d'investisseurs informés indexés par $\mathrm{j} \in[0,1]$, la demande agrégée de ces investisseurs, notée $Z$, est donnée par :

$$
\begin{aligned}
& Z^{\prime}=\int_{0}^{1} Z_{j} d_{j}=\int_{0}^{1} r_{j} \frac{f_{j} R P}{V} d_{j}=\int_{0}^{1} r_{j} \frac{B_{0}+B_{1} Y_{j}+B_{2} P-R P}{V} d_{j}=r \frac{B_{0^{+}} B_{1} \tilde{C}_{+} B_{2} P-R P}{V} \\
& \text { avec } \int_{0}^{1} r_{j} \frac{B_{1} Y_{j}}{V} d_{j}=\int_{0}^{1} r_{j} \frac{B_{1} \tilde{C}}{V} d_{j}+\int_{0}^{1} r_{j} \frac{B_{1} \tilde{e}_{j}}{V} d_{j}=r \frac{B_{1} \tilde{C}}{V} \text { car }: \int_{0}^{1} r_{j} \tilde{e}_{j} d_{j}=0 \text { et } \int_{0}^{1} r_{j} d_{j}=r
\end{aligned}
$$

où $r$ représente la mesure agrégée de tolérance au risque.

À l'équilibre, la demande des investisseurs informés (per capita) égale l'offre nette stochastique : $Z=\tilde{S}$. L'équation (A5) peut alors être résolue pour donner le prix d'équilibre de l'actif risqué.

$$
P=\frac{r B_{0}}{r\left(R-B_{2}\right)}+\frac{B_{1}}{R-B_{2}} \tilde{C}-\frac{V}{r\left(R-B_{2}\right)} \tilde{S}
$$

L'équation (A7) lie le prix d'équilibre de l'actif risqué à sa valeur de fin de période et à l'offre nette du titre. Elle constitue le résultat de base des modèles d'ÉARB. Les formes réduites des paramètres $B_{0}, B_{1}, B_{2}$ et $V$ sont dérivées dans Hellwig (1980):

$$
\begin{aligned}
& B_{0}=\frac{r B_{1} \sigma_{e}^{2} \bar{s}}{\sigma_{e}^{2} \sigma_{s}^{2}+r^{2}}+\left(\frac{\sigma_{e}^{2} \sigma_{s}^{2}}{\sigma_{e}^{2} \sigma_{s}^{2}+r^{2}}-B_{1}\right) \bar{C} \\
& B_{1}=\frac{\sigma_{c}^{2} \sigma_{e}^{2} \sigma_{s}^{2}}{\left(\sigma_{c}^{2}+\sigma_{e}^{2}\right) \sigma_{e}^{2} \sigma_{s}^{2}+r^{2} \sigma_{c}^{2}} \\
& B_{2}=\frac{r^{2} R}{\sigma_{e}^{2} \sigma_{s}^{2}+r^{2}}
\end{aligned}
$$




\subsection{Anticipation moyenne, dispersion des anticipations et prix des titres}

Anticipation moyenne : Dans le cadre d'un modèle d'ÉARB, l'anticipation moyenne des investisseurs informés correspond à la moyenne (entre les investisseurs) des espérances conditionnelles du prix de l'actif risqué étant donnés une information privée et le prix de l'actif risqué :

$$
A M(\tilde{C})=\int_{0}^{1} E\left(\tilde{C} \mid Y_{j}, P\right) d_{j}=\int_{0}^{1}\left(B_{0}+B_{1} Y_{j}+B_{2} P\right) d_{j}
$$

Il est donc possible d'exprimer l'anticipation moyenne des investisseurs informés comme une fonction linéaire du prix actuel et du prix futur de l'actif risqué.

$$
A M(\tilde{C})=B_{0}+B_{1} \tilde{C}+B_{2} P
$$

Dispersion : De la même façon, il est possible d'exprimer la dispersion des anticipations comme étant l'écart type de la distribution des anticipations individuelles.

$$
D / S P(\tilde{C})=\left[\int_{0}^{1}\left(f_{j}-A M\right)^{2} d_{j}\right]^{\frac{1}{2}}
$$

$D I S P$ peut être exprimée en fonction du paramètre $B_{l}$ :

$$
D / S P(\tilde{C})=\left[\int_{0}^{1}\left(\left[B_{0}+B_{1} Y_{j}+B_{2} P\right]-\left[B_{0}+B_{1} \tilde{C}+B_{2} P\right]\right)^{2} d_{j}\right]^{\frac{1}{2}}-\left[\int_{0}^{1}\left(B_{1} e_{j}\right)^{2} d_{j}\right]^{\frac{1}{2}}=B_{1} \sigma_{\theta}
$$

L'équation (A3) permet d'exprimer $B_{0}$ en fonction de l'anticipation moyenne. En substituant cette expression dans (2), le prix d'équilibre de l'actif risqué devient :

$$
P=\frac{r\left(A M-B_{1} \tilde{C}-B_{2} P\right)}{r\left(R-B_{2}\right)}+\frac{B_{1}}{\left(R-B_{2}\right)} \tilde{C}+\frac{V}{r\left(R-B_{2}\right)} \tilde{S}
$$

Il suffit alors de mettre $\mathrm{P}$ en facteur et de l'isoler : 


$$
\begin{aligned}
& \frac{R P}{R-B_{2}}=\frac{r\left(A M-B_{1} \tilde{C}\right)}{r\left(R-B_{2}\right)}+\frac{B_{1}}{R-B_{2}} \tilde{C}-\frac{V}{r\left(R-B_{2}\right)} \tilde{S} \\
& P=\frac{A M}{R}-\frac{V \tilde{S}}{r R}
\end{aligned}
$$

En substituant la valeur de $V$ dans $\mathrm{A} 11$, puis de DISP dans A15, A18 devient :

$$
P=\frac{A M}{R}-\frac{B_{1} \sigma_{e}^{2}}{r R} \tilde{S}=\frac{A M}{R}-\frac{\sigma_{e} \tilde{S} D / S P}{r R}
$$

Nous noterons $\quad \tilde{\boldsymbol{u}}=\tilde{S}-\bar{S}$ l' offre nette aléatoire $\left(\tilde{\boldsymbol{u}} \sim N\left(0, \sigma_{s}\right)\right)$ qui empêche le système de prix de révéler parfaitement l'information. II est donc maintenant possible de transformer l'équation $A 7$, pour obtenir une nouvelle expression du prix d'équilibre de l'actif risqué :

$$
P=\frac{A M}{R}-\left(\frac{\tilde{S} \sigma_{e}}{r R}\right) D I S P=\frac{A M}{R}-\left(\frac{\bar{S} \sigma_{e}}{r R}\right) D I S P-\left(\frac{\tilde{u} \sigma_{e}}{r R}\right) D I S P
$$

Le prix d'équilibre de l'actif risqué est donc relié positivement à l'anticipation moyenne des investisseurs ( $A M)$ et négativement à la dispersion des anticipations individuelles (DISP).

1.3 Détermination d'une expression testable empiriquement : l'équation (A20) est transformée de manière à obtenir une expression où la variable expliquée est le rendement. La différence entre $P_{t}-P_{t-1}$, les prix observés aux temps t et t-1 est égale à :

$$
P_{t^{-}} P_{t-1}=\frac{1}{R}\left(A M_{t}-A M_{t-1}\right)-\frac{\bar{S} \sigma_{e}}{r R} \cdot\left[D / S P_{t^{-}} D / S P_{t-1}\right]-\frac{\sigma_{e}}{r R} \cdot\left[\tilde{u}_{t} D / S P_{t^{-}} \tilde{u}_{t-1} D / S P_{t-1}\right]
$$

Le modèle économétrique pourrait être le suivant :

$$
P_{t}-P_{t-1}=\beta_{0}^{*}+\beta_{1} \Delta A M+\beta_{2} \Delta D I S P+\varepsilon^{*}
$$

ou si l'on normalise les variables par le prix de début de période, pour exprimer le terme de gauche sous la forme d'un rendement :

$$
\frac{P_{t}-P_{t-1}}{P_{t-1}}=R_{t}=\beta_{0}+\beta_{1} \frac{\Delta A M}{P_{t-1}}+\beta_{2} \frac{\Delta D / S P}{P_{t-1}}+\varepsilon_{t}
$$

où $\triangle A M$ et $\triangle D I S P$ représentent respectivement la révision dans l'anticipation moyenne et le changement dans la dispersion des anticipations des agents. 